CRYSTALLOGRAPHIC COMMUNICATIONS

ISSN 2056-9890

Received 8 July 2021

Accepted 17 September 2021

Edited by G. Diaz de Delgado, Universidad de Los Andes, Venezuela

Keywords: crystal structure; density functional theory; quinoxaline; hydrogen bond; Hirshfeld surface analysis.

CCDC reference: 2110486

Supporting information: this article has supporting information at journals.iucr.org/e

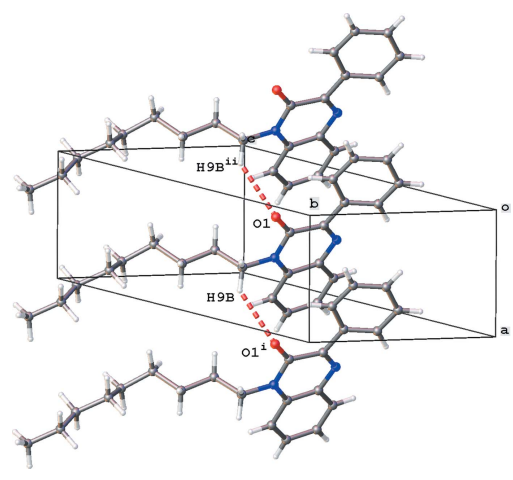

OPEN $\odot$ ACCESS

\section{Crystal structure, Hirshfeld surface analysis and density functional theory study of 1-nonyl-3- phenylquinoxalin-2-one}

\author{
Nadeem Abad, ${ }^{\mathrm{a}, \mathrm{b}}$ Karim Chkirate, ${ }^{\mathrm{a}}$ Fares Hezam Al-Ostoot, ${ }^{\mathrm{b} *}$ Luc Van Meervelt, ${ }^{\mathrm{c}}$ \\ Sanae Lahmidi, ${ }^{a}$ Souad Ferfra, ${ }^{a}$ Youssef Ramli ${ }^{\mathrm{d}}$ and El Mokhtar Essassi ${ }^{\mathrm{a}}$
}

\begin{abstract}
aLaboratory of Heterocyclic Organic Chemistry URAC 21, Pharmacochemistry Competence Center, Av. Ibn Battouta, BP 1014, Faculty of Sciences, Mohammed V University, Rabat, Morocco, ${ }^{\mathbf{b}}$ Department of Biochemistry, Faculty of Education \& Science, AlBaydha University, Yemen, ${ }^{\mathrm{C}} \mathrm{KU}$ Leuven, Chemistry Department, Celestijnenlaan 200F box 2404, Leuven (Heverlee), B-3001, Belgium, and ' Laboratory of Medicinal Chemistry, Drug Sciences Research Center, Faculty of, Medicine and Pharmacy, Mohammed V University in Rabat, Morocco. *Correspondence e-mail: faresalostoot@gmail.com
\end{abstract}

In the title molecule, $\mathrm{C}_{23} \mathrm{H}_{28} \mathrm{~N}_{2} \mathrm{O}$, the phenyl ring is inclined to the quinoxaline ring system at a dihedral angle of $20.40(9)^{\circ}$. In the crystal, $\mathrm{C}-\mathrm{H} \cdots \mathrm{O}$ interactions between neighbouring molecules form chains along the $a$-axis direction. Hirshfeld surface analysis indicates that the most important contributions to the crystal packing are from $\mathrm{H} \cdots \mathrm{H}(70.6 \%), \mathrm{H} \cdots \mathrm{C} / \mathrm{C} \cdots \mathrm{H}$ $(15.5 \%)$ and $\mathrm{H} \cdots \mathrm{O} / \mathrm{O} \cdots \mathrm{H}(4.6 \%)$ interactions. The optimized structure calculated using density functional theory at the B3LYP/6-311 G(d,p) level is compared with the experimentally determined structure in the solid state. The calculated highest occupied molecular orbital (HOMO) and lowest unoccupied molecular orbital (LUMO) energy gap is $3.8904 \mathrm{eV}$. Part of the $n$-nonyl chain attached to one of the nitrogen atoms of the quinoxaline ring system shows disorder and was refined with a double conformation with occupancies of 0.604 (11) and 0.396 (11).

\section{Chemical context}

Nitrogen-based structures have attracted increased attention in structural and inorganic chemistry in recent years because of their interesting properties (Chkirate et al., 2019, 2020a,b, 2021, 2022; Bouzian et al., 2021). The family of quinoxalines, particularly those containing the quinoxalin-2-one moiety, is important in medicinal chemistry because of their wide range of pharmacological applications, including their use as antitumor active agents (Galal et al., 2014), and their antimicrobial (Carta et al., 2003) and biological (Carta et al., 2002) activity. In particular, 3-phenylquinoxaline derivatives are used as anticancer drugs (Abad, Sallam et al., 2021). They also have antifolate activities (Corona et al., 2008). Given the wide range of therapeutic applications for such compounds, and in a continuation of the work already carried out on the synthesis of compounds resulting from quinoxalin-2-one (Al Ati et al., 2021), a similar approach gave the title compound, 1-nonyl-3phenylquinoxalin-2-one $\mathrm{C}_{23} \mathrm{H}_{28} \mathrm{~N}_{2} \mathrm{O}$, (I). Besides the synthesis, we also report the molecular and crystal structures along with a Hirshfeld surface analysis and a density functional theory computational calculation carried out at the B3LYP/6311G(d,p) level. 


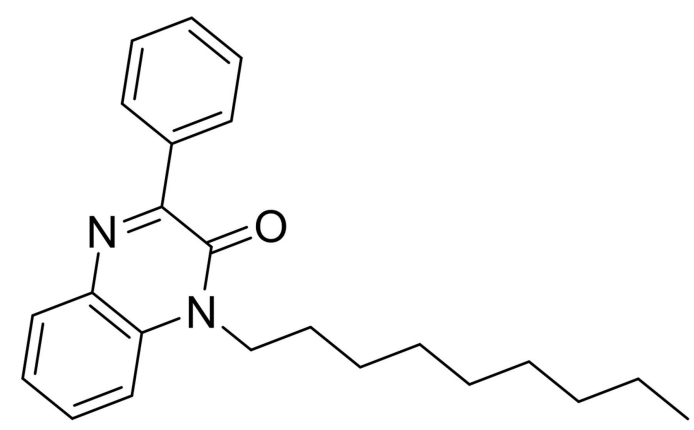

\section{Structural commentary}

The title compound crystallizes in the triclinic space group $P \overline{1}$ with one molecule in the asymmetric unit (Fig. 1). The molecule is not planar, as indicated by the torsion angles $\mathrm{C} 1-$ $\mathrm{C} 2-\mathrm{C} 18-\mathrm{C} 23 \quad\left[-18.6(3)^{\circ}\right]$ and $\mathrm{N} 2-\mathrm{C} 2-\mathrm{C} 18-\mathrm{C} 19$ $\left[-17.3(3)^{\circ}\right]$. The best plane of the phenyl ring C18-C23 (r.m.s. deviation $=0.006 \AA$ ) makes a dihedral angle of $20.40(9)^{\circ}$ with the best plane of the quinoxaline ring system N1/C1/C2/N2/ C3-C8 (r.m.s. deviation $=0.029 \AA$ ). This allows two intramolecular interactions $\mathrm{C} 23-\mathrm{H} 23 \cdots \mathrm{O} 1$ and $\mathrm{C} 19-\mathrm{H} 19 \cdots \mathrm{N} 2$ (Table 1). The $n$-nonyl chain attached to one of the nitrogen atoms of the quinoxaline ring system shows disorder and was refined with a double conformation for atoms $\mathrm{C} 13$ to $\mathrm{C} 16$ with occupancies of 0.604 (11) for $\mathrm{C} 12 A-\mathrm{C} 16 A$ and 0.396 (11) for $\mathrm{C} 12 B-\mathrm{C} 16 B$. The $n$-nonyl chain of set $A$ (starting from C9) has a $a p, a p, a p,+s c, a p, a p, a p$ conformation, while for set $B$ the conformation can be describes as ap, ap, ap, $-s c, a p,-s c, a p$.

\section{Supramolecular features and Hirshfeld surface analysis}

The crystal packing is characterized by $\mathrm{C} 9-\mathrm{H} 9 B \cdots \mathrm{O} 1$ interactions [Fig. $2 ; \mathrm{H} 9 B \cdots \mathrm{O} 1^{\mathrm{i}}=2.772 \AA$; symmetry code: (i) $1+x$, $y, z]$ resulting in ribbon formation in the $a$-axis direction.

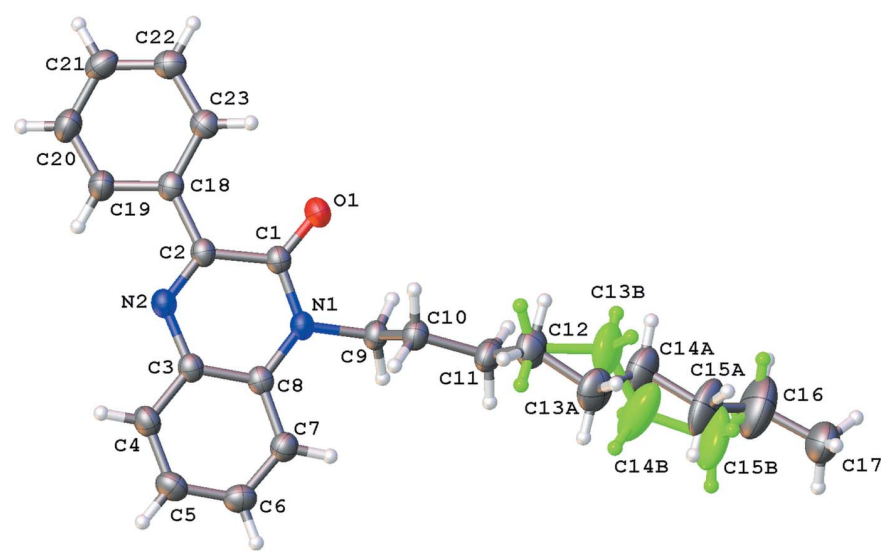

Figure 1

Molecular structure of the title compound with the atom-labelling scheme and ellipsoids drawn at the $50 \%$ probability level. The disordered component of the $n$-nonyl chain with occupancy 0.396 (11) is shown in green.
Table 1

Hydrogen-bond geometry $\left(\AA,^{\circ}\right)$.

\begin{tabular}{lllll}
\hline$D-\mathrm{H} \cdots A$ & $D-\mathrm{H}$ & $\mathrm{H} \cdots A$ & $D \cdots A$ & $D-\mathrm{H} \cdots A$ \\
\hline $\mathrm{C} 19-\mathrm{H} 19 \cdots \mathrm{N} 2$ & 0.93 & 2.44 & $2.758(3)$ & 100 \\
$\mathrm{C} 23-\mathrm{H} 23 \cdots \mathrm{O} 1$ & 0.93 & 2.21 & $2.832(3)$ & 123 \\
\hline
\end{tabular}

Parallel ribbons show short $\mathrm{C} 9-\mathrm{H} 9 A \cdots \mathrm{O} 1$ contacts [Fig. 3; $\mathrm{H} 9 A \cdots \mathrm{O} 1^{\mathrm{ii}}=2.899 \AA$ А̊ symmetry code: (ii) $\left.1-x, 1-y, 1-z\right]$. The crystal packing shows layers of $n$-nonyl chains parallel to the (110) plane with layers of rings in between. Despite the presence of aromatic rings, the packing shows no $\mathrm{C}-\mathrm{H} \cdots \pi$ or $\pi-\pi$ interactions [the shortest centroid-centroid distance is 3.8945 (15) $\AA$ for rings N1/N2/C1-C3/C8 and C18-C23]. The unit cell contains no residual solvent-accessible voids.

The CrystalExplorer program (Turner et al., 2017) was used to further investigate and visualize the intermolecular interactions of (I). The Hirshfeld surfaces for the major and minor occupancy components plotted over $d_{\text {norm }}$ are shown in Fig. 4 . The Hirshfeld surface of the major component (Fig. $4 a$ ) is dominated by white regions representing contacts equal to the van der Waals separation and shows only one red spot (close contacts with a negative $d_{\text {norm }}$ value) indicative of a $\mathrm{H} 16 B \cdots \mathrm{H} 16 B^{\mathrm{iii}}$ contact $[1.995 \AA$; symmetry code: (iii) $2-x$, $2-y, 2-z]$. A similar observation is made for the minor component (Fig. $4 b$ ) where the tiny red spot represents a $\mathrm{H} 15 B \cdots \mathrm{H} 13 B^{\mathrm{i}}$ contact $(2.316 \AA)$.

The overall two-dimensional fingerprint plots (McKinnon et $a l ., 2007)$ for the two components are shown in Fig. $5 a$ and $b$, while those delineated into $\mathrm{H} \cdots \mathrm{H}$ and $\mathrm{H} \cdots \mathrm{C} / \mathrm{C} \cdots \mathrm{H}$ contacts are illustrated in Fig. $5 c-f$, respectively, together with their

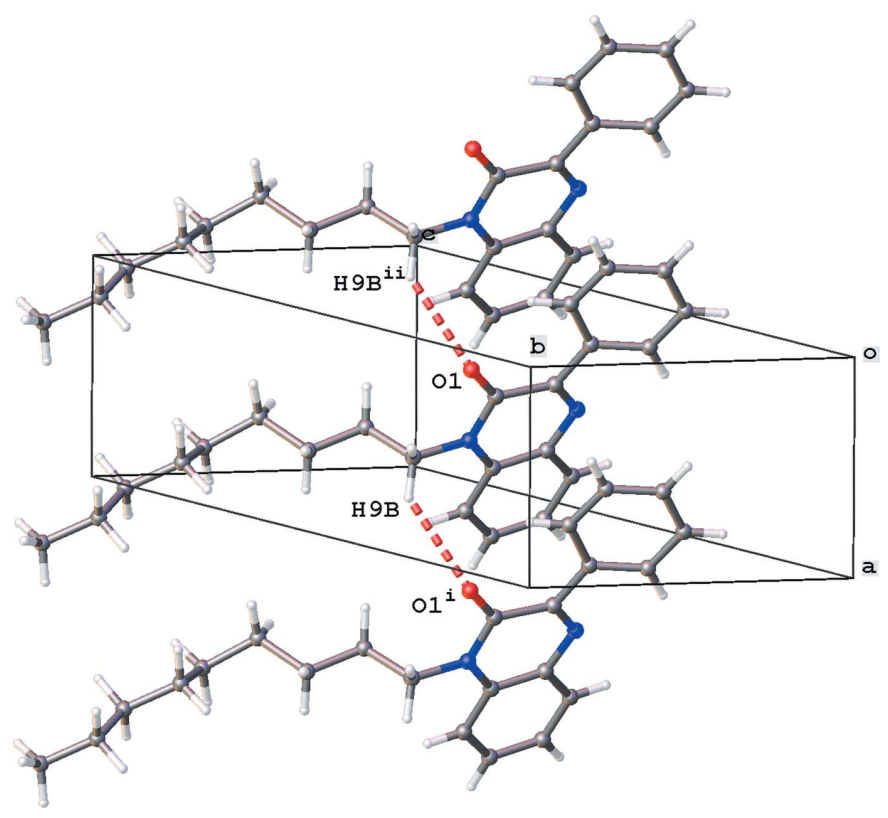

Figure 2

Partial view of the crystal packing of the title compound showing the $\mathrm{C}-$ $\mathrm{H} \cdots \mathrm{O}$ interaction (red dashed lines) and chain formation in the $a$-axis direction. Only the major component of the $n$-nonyl chain is shown. Symmetry codes: (i) $1+x, y, z$; (ii) $-1+x, y, z$. 


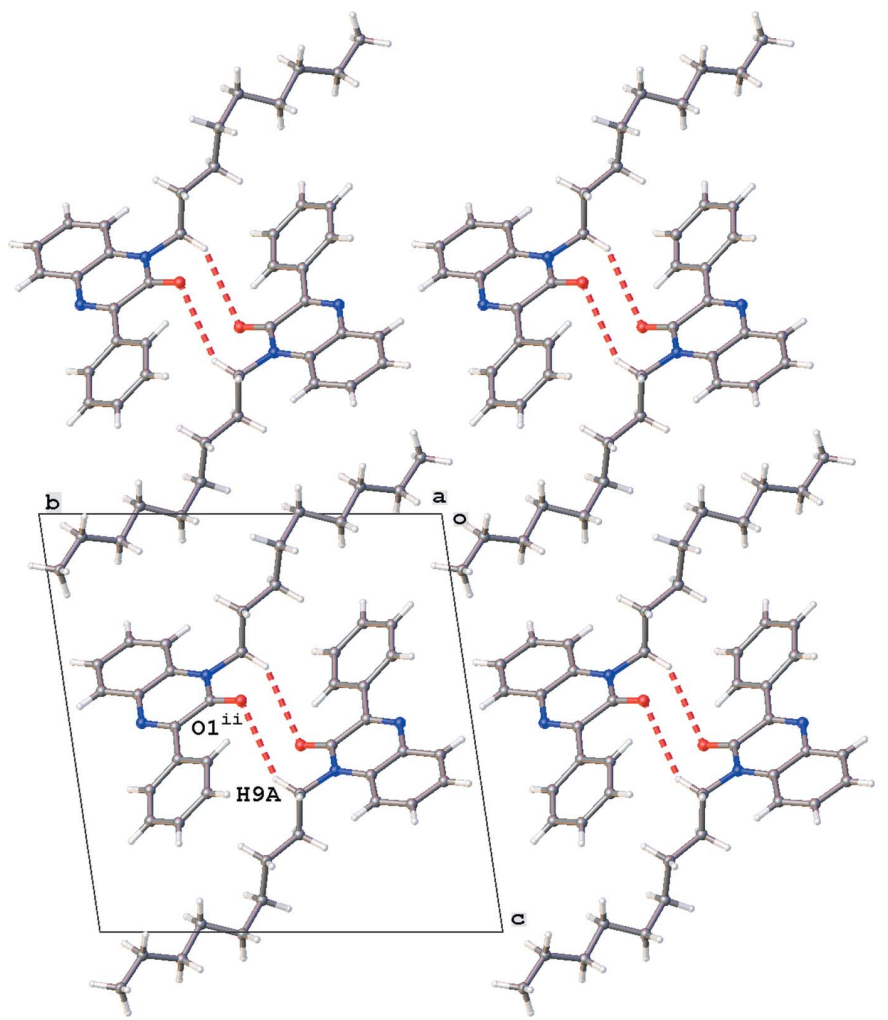

Figure 3

A view down the $a$ axis of the crystal packing of the title compound showing the alternating layers of $n$-octyl chains and aromatic rings. Only the major disorder component of the $n$-nonyl chain is shown.

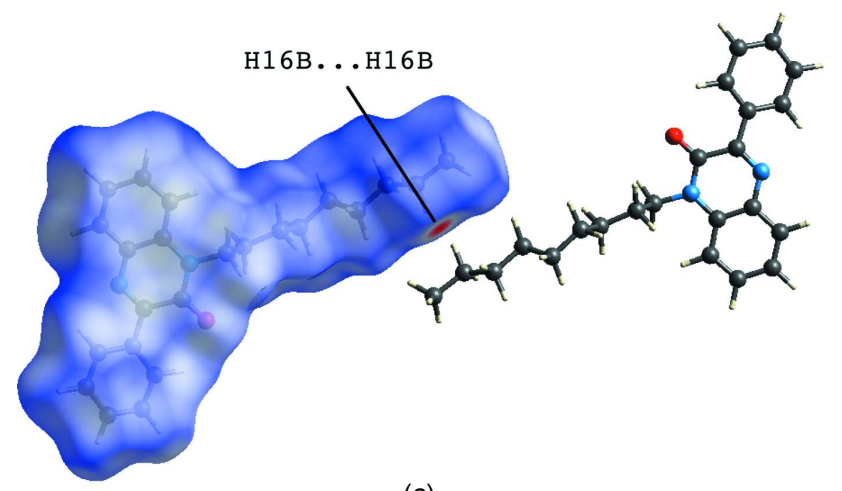

(a)

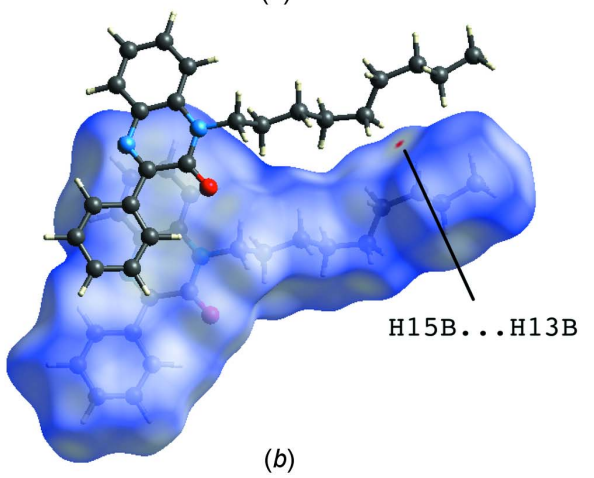

Figure 4

View of the three-dimensional Hirshfeld surface plotted over $d_{\text {norm }}$ for $(a)$ the major component (range -0.3582 to 1.3718 a.u.) and (b) the minor component (range -0.0395 to 1.5398 a.u.) of the title compound. relative contributions to the Hirshfeld surface. The most important interaction is $\mathrm{H} \cdots \mathrm{H}$, contributing $70.6 \%$ (major component) or $70.5 \%$ (minor component) to the overall crystal packing, which is reflected in Fig. $5 c$ and $d$ as widely scattered points of high density due to the large hydrogen content of the molecule, with a sharp tip at $d_{\mathrm{e}}=d_{\mathrm{i}}=0.87 \AA$ in the case of the major component. The second most important are $\mathrm{C}-\mathrm{H}$ interactions, contributing $15.5 \%$ (major component) or $15.6 \%$ (minor component), for which the fingerprint plot (Fig. $5 e$ and $f$ ) shows characteristic wings with tips at $d_{e}+d_{\mathrm{i}} \simeq$ $2.80 \AA$ A. Other contacts contribute only $4.6 \%(\mathrm{H} \cdots \mathrm{O} / \mathrm{O} \cdots \mathrm{H})$, $4.3 \%(\mathrm{C} \cdots \mathrm{C}), 2.4 \%(\mathrm{H} \cdots \mathrm{N} / \mathrm{N} \cdots \mathrm{H}), 2.2 \%(\mathrm{~N} \cdots \mathrm{C} / \mathrm{C} \cdots \mathrm{N})$, $0.3 \%(\mathrm{O} \cdots \mathrm{O})$ and $0.1 \%(\mathrm{O} \cdots \mathrm{C} / \mathrm{C} \cdots \mathrm{O})$ to the Hirshfeld surface.

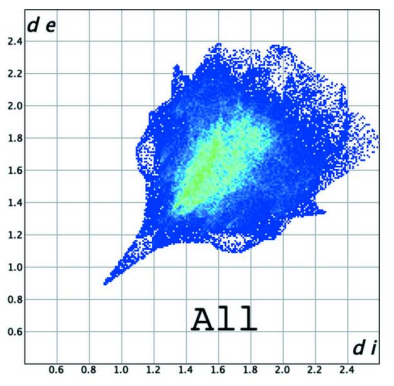

(a)

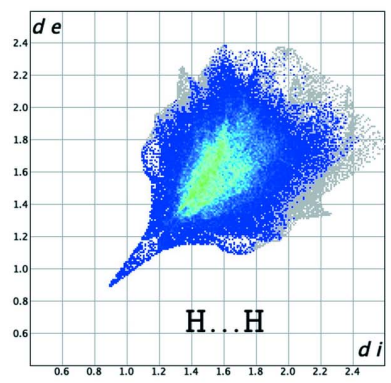

(c)

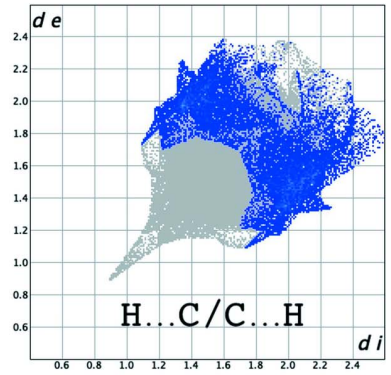

(e)

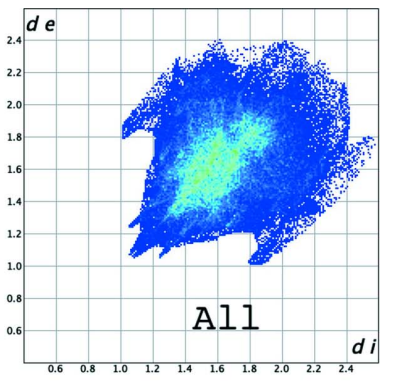

(b)

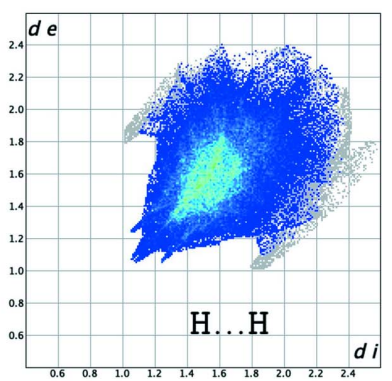

(d)

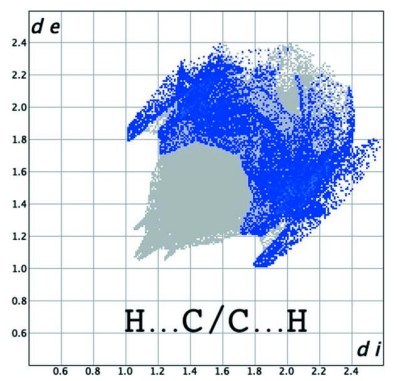

$(f)$
Figure 5

The full two-dimensional fingerprint plots showing $(a, b)$ all interactions, and delineated into $(c, d) \mathrm{H} \cdots \mathrm{H}$ and $(e, f) \mathrm{H} \cdots \mathrm{C} / \mathrm{C} \cdots \mathrm{H}$ interactions for the major (left) and minor (right) component of the title compound. The $d_{\mathrm{i}}$ and $d_{\mathrm{e}}$ values are the closest internal and external distances (in $\AA$ ) from points on the Hirshfeld surface. 
Table 2

Comparison (X-ray and density functional theory) of selected bond lengths and angles $\left(\AA{ }^{\circ}\right)$.

\begin{tabular}{lll}
\hline & X-ray & B3LYP/6-311G(d,p) \\
\hline $\mathrm{O} 1-\mathrm{C} 1$ & $1.221(3)$ & 1.2236 \\
$\mathrm{~N} 1-\mathrm{C} 1$ & $1.379(3)$ & 1.3975 \\
$\mathrm{~N} 1-\mathrm{C} 8$ & $1.387(3)$ & 1.3892 \\
$\mathrm{~N} 1-\mathrm{C} 9$ & $1.474(3)$ & 1.4735 \\
$\mathrm{~N} 2-\mathrm{C} 2$ & $1.296(3)$ & 1.299 \\
$\mathrm{~N} 2-\mathrm{C} 3$ & $1.384(3)$ & 1.3723 \\
$\mathrm{C} 2-\mathrm{C} 18$ & $1.481(3)$ & 1.4862 \\
$\mathrm{C} 1-\mathrm{N} 1-\mathrm{C} 8$ & $122.74(19)$ & 122.5778 \\
$\mathrm{C} 1-\mathrm{N} 1-\mathrm{C} 9$ & $116.64(19)$ & 116.1328 \\
$\mathrm{C} 8-\mathrm{N} 1-\mathrm{C} 9$ & $120.60(19)$ & 121.2682 \\
$\mathrm{O} 1-\mathrm{C} 1-\mathrm{N} 1$ & $120.6(2)$ & 120.2255 \\
$\mathrm{O} 1-\mathrm{C} 1-\mathrm{C} 2$ & $124.1(2)$ & 124.5602 \\
$\mathrm{~N} 1-\mathrm{C} 1-\mathrm{C} 2$ & $115.22(19)$ & 115.2104 \\
$\mathrm{C} 2-\mathrm{N} 2-\mathrm{C} 3$ & $120.3(2)$ & 121.844 \\
$\mathrm{~N} 2-\mathrm{C} 2-\mathrm{C} 1$ & $122.0(2)$ & 117.4937 \\
$\mathrm{~N} 2-\mathrm{C} 2-\mathrm{C} 18$ & $117.6(2)$ & 118.5343 \\
$\mathrm{~N} 2-\mathrm{C} 3-\mathrm{C} 4$ & $118.7(2)$ & 121.9008 \\
$\mathrm{~N} 2-\mathrm{C} 3-\mathrm{C} 8$ & $121.6(2)$ & 117.4153 \\
$\mathrm{~N} 1-\mathrm{C} 8-\mathrm{C} 3$ & $117.6(2)$ & 123.4308 \\
$\mathrm{~N} 1-\mathrm{C} 8-\mathrm{C} 7$ & $123.5(2)$ & 112.9655 \\
$\mathrm{~N} 1-\mathrm{C} 9-\mathrm{C} 10$ & $112.61(19)$ & \\
\hline
\end{tabular}

\section{Density functional theory calculations}

The structure in the gas phase of the title compound was optimized by means of density functional theory. The density functional theory calculation was performed by the hybrid B3LYP method and the 6-311 G(d,p) basis-set, which is based on Becke's model (Becke, 1993) and considers a mixture of the exact (Hartree-Fock) and density functional theory exchange utilizing the $\mathrm{B} 3$ functional, together with the LYP correlation functional (Lee et al., 1988). After obtaining the converged geometry, the harmonic vibrational frequencies were calculated at the same theoretical level to confirm that the number of imaginary frequencies is zero for the stationary point. Both the geometry optimization and harmonic vibrational frequency analysis of the title compound were performed with the GAUSSIAN 09 program (Frisch et al., 2009). Theoretical and experimental results related to bond lengths and angles, which are in good agreement, are summarized in Table 2. Calculated numerical values for the title compound, including electronegativity $(\chi)$, hardness $(\eta)$, ionization potential $(I)$, dipole moment $(\mu)$, electron affinity $(A)$, electrophilicity $(\omega)$ and softness $(\sigma)$, are collated in Table 3. The electron transition from the highest occupied molecular orbital (HOMO) to the lowest unoccupied molecular orbital (LUMO) energy level is shown in Fig. 6. The HOMO and LUMO are localized in the plane extending over the whole 1-nonyl-3-phenylquinoxalin-2-one system. The energy band gap $\left[\Delta E=E_{\mathrm{LUMO}}-E_{\mathrm{HOMO}}\right]$ of the molecule is $3.8904 \mathrm{eV}$, and the frontier molecular orbital energies, $E_{\mathrm{HOMO}}$ and $E_{\mathrm{LUMO}}$, are -6.1155 and $-2.2251 \mathrm{eV}$, respectively.

\section{Database survey}

A search of the Cambridge Structural Database (CSD version 5.42, updated May 2021; Groom et al., 2016) for the quinox-
Table 3

Calculated energies.

\begin{tabular}{lr}
\hline Molecular Energy & Compound (I) \\
\hline Total Energy, $T E(\mathrm{eV})$ & -29343.5617 \\
$E_{\mathrm{HOMO}}(\mathrm{eV})$ & -6.1155 \\
$E_{\mathrm{LUMO}}(\mathrm{eV})$ & -2.2251 \\
Gap, $\Delta E(\mathrm{eV})$ & 3.8904 \\
Dipole moment, $\mu$ (Debye) & 3.0783 \\
Ionization potential, $I(\mathrm{eV})$ & 6.1155 \\
Electron affinity, $A$ & 2.2251 \\
Electronegativity, $\chi$ & 4.1703 \\
Hardness, $\eta$ & 1.9452 \\
Electrophilicity index, $\omega$ & 4.4703 \\
Softness, $\sigma$ & 0.5141 \\
Fraction of electron transferred, $\Delta N$ & 0.7274 \\
\hline
\end{tabular}

alin-2(1H)-one fragment yielded multiple matches (180 hits). Of these, three compounds had an alkyl substituent on N1 and a phenyl ring on $\mathrm{C} 2$ comparable to (I) and are shown in Fig. 7. The first two compounds carry an ethyl [(II), refcode MAGBIJ; Al Ati et al., 2021] or methyl [(III), refcode BUDMAP; Benzeid et al., 2009] on N1. The third one [(IV), refcode ASAZEC; Abad, Ferfra et al., 2021] has an $n$-octyl chain on $\mathrm{N} 1$ instead of a $n$-nonyl chain. The phenyl ring in MAGBIJ is inclined to the quinoxaline ring system by
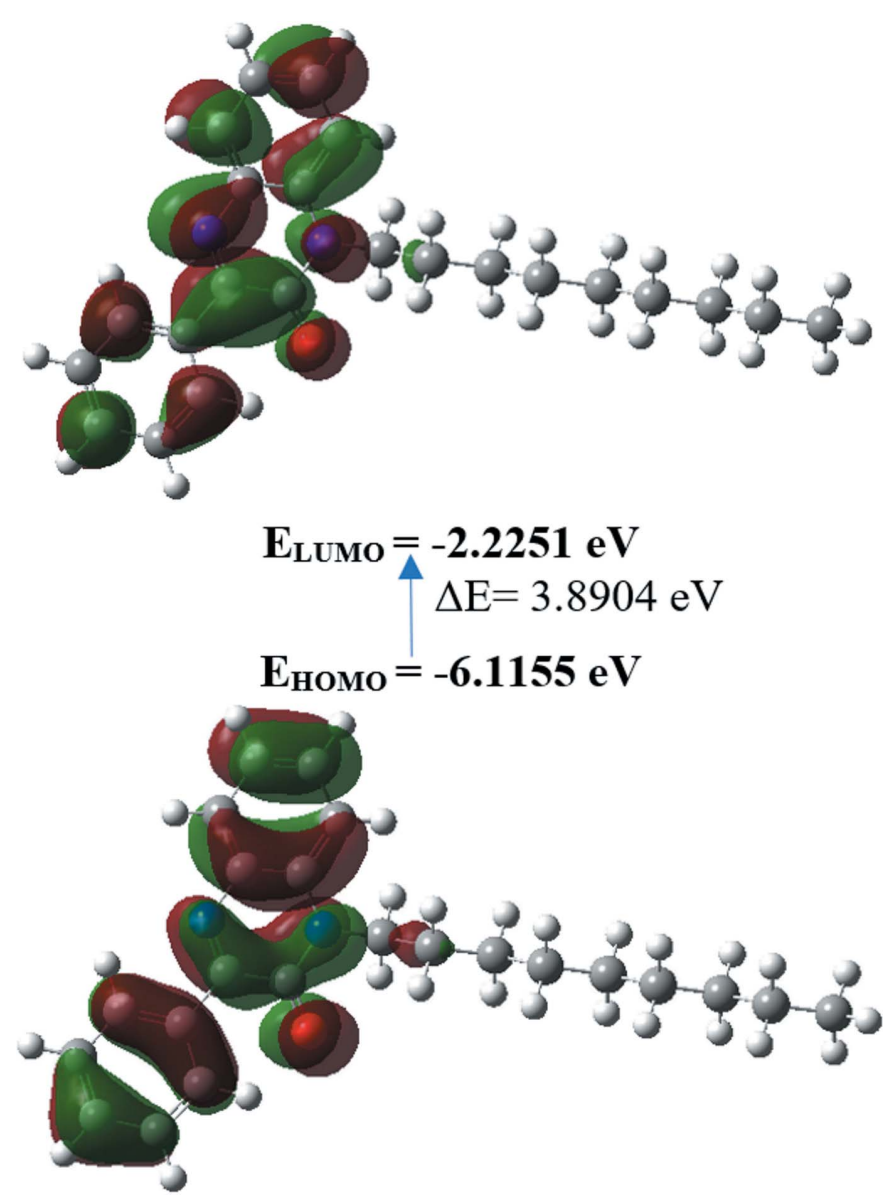

Figure 6

HOMO-LUMO and the energy band gap of the title compound. 
<smiles>CCn1c(=O)c(-c2ccccc2)nc2ccccc21</smiles>

II

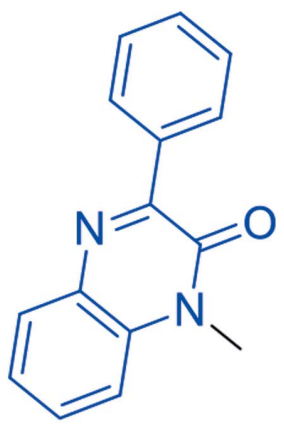

III

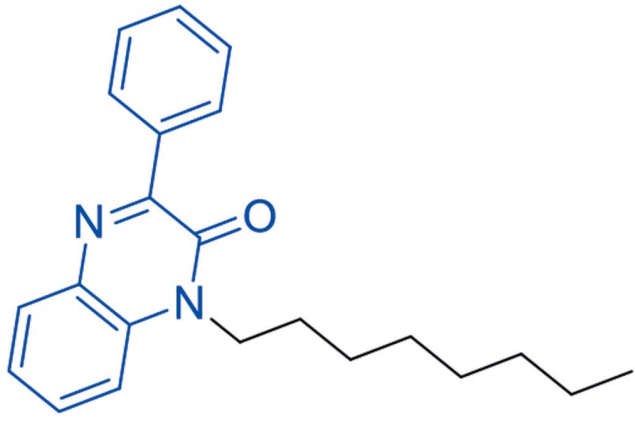

IV

Figure 7

Structures similar to (I): (II) (CSD refcode MAGBIJ), (III) (CSD refcode BUDMAP) and (IV) (CSD refcode ASAZEC) obtained during the database search. The search fragment is indicated in blue.

$25.81(12)^{\circ}$. For BUDMAP, the dihedral angles are 19.3 (1) and $30.4(1)^{\circ}$ for the two molecules present in the asymmetric unit. For ASAZEC, the dihedral angle is $12.90(4)^{\circ}$ and no disorder is observed in the $n$-octyl chain, which could be the consequence of the data collection being undertaken at 150 (2) K. Despite the similarity to the title compound, ASAZEC crystallizes in space group $C 2 / c$ and the molecules are linked by $\mathrm{C}-\mathrm{H} \cdots \pi$ interactions and form stacks in the $b$ axis direction.

\section{Synthesis and crystallization}

To a solution of 3-phenylquinoxalin-2(1H)-one $(0.5 \mathrm{~g}$, $2.25 \mathrm{mmol})$ in dichloromethane $(20 \mathrm{ml})$ were added 1-chlorononane $(0.2 \mathrm{ml}, 2.25 \mathrm{mmol})$, sodium hydroxide $(0.1 \mathrm{~g}$, $2.25 \mathrm{mmol}$ ) and a catalytic quantity of tetra- $n$-butylammonium bromide. The reaction mixture was stirred at room temperature for $24 \mathrm{~h}$. The solution was filtered and the solvent removed under reduced pressure. The residue thus obtained was chromatographed on a silica gel column using a hexane/ ethyl acetate 9:1 mixture as eluent. The solid obtained was recrystallized from ethanol to afford colourless crystals (yield: $70 \%) .{ }^{1} \mathrm{H}$ NMR $\left(300 \mathrm{MHz}, \mathrm{CDCl}_{3}\right) \delta \mathrm{ppm}: 0.89\left(t, 3 \mathrm{H}, \mathrm{CH}_{3}, J=\right.$ $6 \mathrm{~Hz}) ; 1.19-1.42\left(m, 12 \mathrm{H}, \mathrm{CH}_{2}\right) ; 1.65-1.76$ (quin, $2 \mathrm{H}, \mathrm{N}-$ $\left.\mathrm{CH}_{2}-\mathrm{CH}_{2}\right) ; 4.20\left(t, 2 \mathrm{H}, \mathrm{N}-\mathrm{CH}_{2}, J=6 \mathrm{~Hz}\right) ; 7.22-8.24(m, 9 \mathrm{H}$, $\left.\mathrm{CH}_{\text {arom }}\right) ;{ }^{13} \mathrm{C} \mathrm{NMR}\left(75 \mathrm{MHz}, \mathrm{CDCl}_{3}\right) \delta$ ppm: $14.12\left(\mathrm{CH}_{3}\right)$; 22.67, 27.11, 27.32, 29.24, 29.36, 29.51, $31.85\left(\mathrm{CH}_{2}\right) ; 42.68(\mathrm{~N}-$ $\left.\mathrm{CH}_{2}\right) ; 113.59,123.49,128.05,129.63,130.22,130.28,130.72$ $\left(\mathrm{CH}_{\text {arom }}\right) ; 132.61,133.42,136.14,154.11\left(\mathrm{C}_{\mathrm{q}}\right) ; 154.40(\mathrm{C}=\mathrm{O})$.

Table 4

Experimental details.

\begin{tabular}{|c|c|}
\hline \multicolumn{2}{|l|}{ Crystal data } \\
\hline Chemical formula & $\mathrm{C}_{23} \mathrm{H}_{28} \mathrm{~N}_{2} \mathrm{O}$ \\
\hline$M_{\mathrm{r}}$ & 348.47 \\
\hline Crystal system, space group & Triclinic, $P \overline{1}$ \\
\hline Temperature $(\mathrm{K})$ & 293 \\
\hline$a, b, c(\AA)$ & $5.2353(2), 13.5065(5), 14.3158(5)$ \\
\hline$\alpha, \beta, \gamma\left({ }^{\circ}\right)$ & $98.045(3), 98.327(3), 91.255(3)$ \\
\hline$V\left(\AA^{3}\right)$ & $990.83(6)$ \\
\hline$Z$ & 2 \\
\hline Radiation type & Mo $K \alpha$ \\
\hline$\mu\left(\mathrm{mm}^{-1}\right)$ & 0.07 \\
\hline Crystal size $(\mathrm{mm})$ & $0.45 \times 0.3 \times 0.15$ \\
\hline \multicolumn{2}{|l|}{ Data collection } \\
\hline Diffractometer & $\begin{array}{l}\text { SuperNova, Single source at offset/ } \\
\text { far, Eos }\end{array}$ \\
\hline Absorption correction & $\begin{array}{l}\text { Multi-scan (CrysAlis PRO; Rigaku } \\
\text { OD, 2018) }\end{array}$ \\
\hline$T_{\min }, T_{\max }$ & $0.686,1.000$ \\
\hline $\begin{array}{l}\text { No. of measured, independent and } \\
\text { observed }[I>2 \sigma(I)] \text { reflections }\end{array}$ & $20242,4058,2864$ \\
\hline$R_{\text {int }}$ & 0.022 \\
\hline$(\sin \theta / \lambda)_{\max }\left(\AA^{-1}\right)$ & 0.625 \\
\hline \multicolumn{2}{|l|}{ Refinement } \\
\hline$R\left[F^{2}>2 \sigma\left(F^{2}\right)\right], w R\left(F^{2}\right), S$ & $0.070,0.240,1.05$ \\
\hline No. of reflections & 4058 \\
\hline No. of parameters & 264 \\
\hline No. of restraints & 70 \\
\hline $\mathrm{H}$-atom treatment & $\mathrm{H}$-atom parameters constrained \\
\hline$\Delta \rho_{\max }, \Delta \rho_{\min }\left(\mathrm{e} \AA^{-3}\right)$ & $0.37,-0.45$ \\
\hline
\end{tabular}

Computer programs: CrysAlis PRO (Rigaku OD, 2018), SHELXT2014/5 (Sheldrick, 2015a), SHELXL2016/4 (Sheldrick, 2015b) and OLEX2 (Dolomanov et al., 2009).

\section{Refinement}

Crystal data, data collection and structure refinement details are given in Table 4. C-bound $\mathrm{H}$ atoms were positioned geometrically $(\mathrm{C}-\mathrm{H}=0.93-0.97 \AA)$ and included as riding contributions with isotropic displacement parameters fixed at 1.2 times $U_{\text {eq }}$ of the parent atoms (1.5 for methyl groups). During the refinement, the difference-Fourier map revealed disorder for atoms $\mathrm{C} 13, \mathrm{C} 14$ and $\mathrm{C} 15$ of the nonyl chain and two conformations were refined with distance restraints (1.512 $\AA$ ) for the $\mathrm{C}-\mathrm{C}$ bonds involved and RIGU restraints for the nonyl chain $\mathrm{C} 11-\mathrm{C} 17$. At the end of the refinement, the occupancy factors of the two components converged to 0.604 (11) and 0.396 (11) and the final difference-Fourier map showed no residual peaks of chemical significance.

\section{Acknowledgements}

Authors' contributions are as follows. Conceptualization, NA; methodology, NA and YR; investigation, $\mathrm{KC}$ and NA; theoretical calculations, $\mathrm{KC}$; writing (original draft), $\mathrm{KC}$ and LVM; writing (review and editing of the manuscript), FHAO; formal analysis, SL and SF; supervision, EME; crystal-structure determination and validation, LVM.

\section{Funding information}

LVM thanks the Hercules Foundation for supporting the purchase of the diffractometer through project AKUL/09/ 0035 . 


\section{References}

Abad, N., Ferfra, S., Essassi, E. M., Mague, J. T. \& Ramli, Y. (2021). Z. Kristallogr. New Cryst. Struct. 236, 173-175.

Abad, N., Sallam, H. H., Al-Ostoot, F. H., Khamees, H. A., Alhoraibi, S. A., Khanum, S. A., Madegowda, M., Hafi, M. E., Mague, J. T., Essassi, E. M. \& Ramli, Y. (2021). J. Mol. Struct. 1232, 130004.

Al Ati, G., Chkirate, K., Mashrai, A., Mague, J. T., Ramli, Y., Achour, R. \& Essassi, E. M. (2021). Acta Cryst. E77, 18-22.

Becke, A. D. (1993). J. Chem. Phys. 98, 5648-5652.

Benzeid, H., Essassi, E. M., Saffon, N., Garrigues, B. \& Ng, S. W. (2009). Acta Cryst. E65, o2323.

Bouzian, Y., Sert, Y., Khalid, K., Van Meervelt, L., Chkirate, K., Mahi, L., Ahabchane, N. H., Talbaoui, A. \& Essassi, E. M. (2021). J. Mol. Struct. 1246, 131217.

Carta, A., Loriga, M., Zanetti, S. \& Sechi, L. A. (2003). Farmaco, 58, 1251-1255.

Carta, A., Sanna, P., Loriga, M., Setzu, M. G., La Colla, P. \& Loddo, R. (2002). Farmaco, 57, 19-25.

Chkirate, K., Akachar, J., Hni, B., Hökelek, T., Anouar, E. H., Talbaoui, A., Mague, J. T., Sebbar, N. K., Ibrahimi, A. \& Essassi, E. M. (2022). J. Mol. Struct. 1247, 131188.

Chkirate, K., Azgaou, K., Elmsellem, H., El Ibrahimi, B., Sebbar, N. K., Anouar, E. H., Benmessaoud, M., El Hajjaji, S. \& Essassi, E. M. (2021). J. Mol. Liq. 321, 114750.

Chkirate, K., Fettach, S., El Hafi, M., Karrouchi, K., Elotmani, B., Mague, J. T., Radi, S., Faouzi, M. E. A., Adarsh, N. N., Essassi, E. M. \& Garcia, Y. (2020a). J. Inorg. Biochem. 208, 21-28.

Chkirate, K., Fettach, S., Karrouchi, K., Sebbar, N. K., Essassi, E. M., Mague, J. T., Radi, S., El Abbes Faouzi, M., Adarsh, N. N. \& Garcia, Y. (2019). J. Inorg. Biochem. 191, 21-28.

Chkirate, K., Karrouchi, K., Dege, N., Kheira Sebbar, N., Ejjoummany, A., Radi, S., Adarsh, N. N., Talbaoui, A., Ferbinteanu, M., Essassi, E. M. \& Garcia, Y. (2020b). New J. Chem. 44, 2210-2221.

Corona, P., Loriga, M., Costi, M. P., Ferrari, S. \& Paglietti, G. (2008). Eur. J. Med. Chem. 43, 189-203.
Dolomanov, O. V., Bourhis, L. J., Gildea, R. J., Howard, J. A. K. \& Puschmann, H. (2009). J. Appl. Cryst. 42, 339-341.

Frisch, M. J., Trucks, G. W., Schlegel, H. B., Scuseria, G. E., Robb, M. A., Cheeseman, J. R., Scalmani, G., Barone, V., Mennucci, B., Petersson, G. A., Nakatsuji, H., Caricato, M., Li, X., Hratchian, H. P., Izmaylov, A. F., Bloino, J., Zheng, G., Sonnenberg, J. L., Hada, M., Ehara, M., Toyota, K., Fukuda, R., Hasegawa, J., Ishida, M., Nakajima, T., Honda, Y., Kitao, O., Nakai, H., Vreven, T., Montgomery, J. A. Jr, Peralta, J. E., Ogliaro, F., Bearpark, M., Heyd, J. J., Brothers, E., Kudin, K. N., Staroverov, V. N., Kobayashi, R., Normand, J., Raghavachari, K., Rendell, A., Burant, J. C., Iyengar, S. S., Tomasi, J., Cossi, M., Rega, N., Millam, J. M., Klene, M., Knox, J. E., Cross, J. B., Bakken, V., Adamo, C., Jaramillo, J., Gomperts, R., Stratmann, R. E., Yazyev, O., Austin, A. J., Cammi, R., Pomelli, C., Ochterski, J. W., Martin, R. L., Morokuma, K., Zakrzewski, V. G., Voth, G. A., Salvador, P., Dannenberg, J. J., Dapprich, S., Daniels, A. D., Farkas, O., Foresman, J. B., Ortiz, J. V., Cioslowski, J. \& Fox, D. J. (2009). GAUSSIAN09. Rev. pp. A02 Gaussian Inc, Wallingford, CT, USA.

Galal, S. A., Khairat, S. H. M., Ragab, F. A. F., Abdelsamie, A. S., Ali, M. M., Soliman, S. M., Mortier, J., Wolber, G. \& El Diwani, H. I. (2014). Eur. J. Med. Chem. 86, 122-132.

Groom, C. R., Bruno, I. J., Lightfoot, M. P. \& Ward, S. C. (2016). Acta Cryst. B72, 171-179.

Lee, C., Yang, W. \& Parr, R. G. (1988). Phys. Rev. B, 37, 785-789.

McKinnon, J. J., Jayatilaka, D. \& Spackman, M. A. (2007). Chem. Commun. pp. 3814-3816.

Rigaku OD (2018). CrysAlis PRO. Rigaku Oxford Diffraction, Yarnton, England.

Sheldrick, G. M. (2015a). Acta Cryst. A71, 3-8.

Sheldrick, G. M. (2015b). Acta Cryst. C71, 3-8.

Turner, M. J., McKinnon, J. J., Wolff, S. K., Grimwood, D. J., Spackman, P. R., Jayatilaka, D. \& Spackman, M. A. (2017). CrystalExplorer17. The University of Western Australia. 


\section{supporting information}

Acta Cryst. (2021). E77, 1037-1042［https://doi.org/10.1107/S2056989021009737]

\section{Crystal structure, Hirshfeld surface analysis and density functional theory study}

\section{of 1-nonyl-3-phenylquinoxalin-2-one}

\section{Nadeem Abad, Karim Chkirate, Fares Hezam Al-Ostoot, Luc Van Meervelt, Sanae Lahmidi, Souad Ferfra, Youssef Ramli and El Mokhtar Essassi}

\section{Computing details}

Data collection: CrysAlis PRO (Rigaku OD, 2018); cell refinement: CrysAlis PRO (Rigaku OD, 2018); data reduction: CrysAlis PRO (Rigaku OD, 2018); program(s) used to solve structure: SHELXT2014/5 (Sheldrick, 2015a); program(s) used to refine structure: SHELXL2016/4 (Sheldrick, 2015b); molecular graphics: OLEX2 (Dolomanov et al., 2009); software used to prepare material for publication: OLEX2 (Dolomanov et al., 2009).

1-Nonyl-3-phenylquinoxalin-2-one

\section{Crystal data}

$\mathrm{C}_{23} \mathrm{H}_{28} \mathrm{~N}_{2} \mathrm{O}$

$M_{r}=348.47$

Triclinic, $P \overline{1}$

$a=5.2353(2) \AA$

$b=13.5065(5) \AA$

$c=14.3158(5) \AA$

$\alpha=98.045(3)^{\circ}$

$\beta=98.327(3)^{\circ}$

$\gamma=91.255(3)^{\circ}$

$V=990.83(6) \AA^{3}$

\section{Data collection}

SuperNova, Single source at offset/far, Eos diffractometer

Radiation source: micro-focus sealed X-ray tube, SuperNova (Mo) X-ray Source

Mirror monochromator

Detector resolution: 15.9631 pixels $\mathrm{mm}^{-1}$

$\omega$ scans

Absorption correction: multi-scan

(CrysAlisPro; Rigaku OD, 2018)

\section{Refinement}

Refinement on $F^{2}$

Least-squares matrix: full

$R\left[F^{2}>2 \sigma\left(F^{2}\right)\right]=0.070$

$w R\left(F^{2}\right)=0.240$

$S=1.05$

4058 reflections
$Z=2$
$F(000)=376$
$D_{\mathrm{x}}=1.168 \mathrm{Mg} \mathrm{m}^{-3}$

Mo $K \alpha$ radiation, $\lambda=0.71073 \AA$

Cell parameters from 6605 reflections

$\theta=3.0-26.4^{\circ}$

$\mu=0.07 \mathrm{~mm}^{-1}$

$T=293 \mathrm{~K}$

Block, colourless

$0.45 \times 0.3 \times 0.15 \mathrm{~mm}$

$T_{\min }=0.686, T_{\max }=1.000$

20242 measured reflections

4058 independent reflections

2864 reflections with $I>2 \sigma(I)$

$R_{\text {int }}=0.022$

$\theta_{\text {max }}=26.4^{\circ}, \theta_{\min }=2.9^{\circ}$

$h=-6 \rightarrow 6$

$k=-16 \rightarrow 16$

$l=-17 \rightarrow 17$

264 parameters

70 restraints

Primary atom site location: dual

Hydrogen site location: inferred from neighbouring sites

$\mathrm{H}$-atom parameters constrained 
$w=1 /\left[\sigma^{2}\left(F_{\mathrm{o}}^{2}\right)+(0.1119 P)^{2}+0.4832 P\right]$

where $P=\left(F_{\mathrm{o}}^{2}+2 F_{\mathrm{c}}{ }^{2}\right) / 3$

$(\Delta / \sigma)_{\max }<0.001$

$$
\Delta \rho_{\max }=0.37 \text { e } \AA^{-3}
$$

$\Delta \rho_{\min }=-0.45$ e $\AA^{-3}$

Special details

Geometry. All esds (except the esd in the dihedral angle between two 1.s. planes) are estimated using the full covariance matrix. The cell esds are taken into account individually in the estimation of esds in distances, angles and torsion angles; correlations between esds in cell parameters are only used when they are defined by crystal symmetry. An approximate (isotropic) treatment of cell esds is used for estimating esds involving 1.s. planes.

Fractional atomic coordinates and isotropic or equivalent isotropic displacement parameters $\left(\AA^{2}\right)$

\begin{tabular}{|c|c|c|c|c|c|}
\hline & $x$ & $y$ & $z$ & $U_{\text {iso }} * / U_{\text {eq }}$ & Occ. $(<1)$ \\
\hline$\overline{\mathrm{O} 1}$ & $0.3177(4)$ & $0.43358(13)$ & $0.55347(14)$ & $0.0685(5)$ & \\
\hline N1 & $0.6712(4)$ & $0.35716(13)$ & $0.61382(14)$ & $0.0498(5)$ & \\
\hline $\mathrm{C} 1$ & $0.4408(5)$ & $0.35784(17)$ & $0.55355(17)$ & $0.0510(5)$ & \\
\hline $\mathrm{N} 2$ & $0.4810(4)$ & $0.17973(14)$ & $0.50005(14)$ & $0.0527(5)$ & \\
\hline $\mathrm{C} 2$ & $0.3592(4)$ & $0.26106(16)$ & $0.49062(16)$ & $0.0489(5)$ & \\
\hline $\mathrm{C} 3$ & $0.6998(5)$ & $0.18102(17)$ & $0.56737(17)$ & $0.0516(6)$ & \\
\hline $\mathrm{C} 4$ & $0.8245(5)$ & $0.09152(19)$ & $0.5763(2)$ & $0.0625(7)$ & \\
\hline $\mathrm{H} 4$ & 0.755288 & 0.032153 & 0.539594 & $0.075^{*}$ & \\
\hline $\mathrm{C} 5$ & $1.0489(6)$ & $0.0911(2)$ & $0.6392(2)$ & $0.0698(7)$ & \\
\hline H5 & 1.131744 & 0.031553 & 0.644889 & $0.084^{*}$ & \\
\hline C6 & $1.1516(5)$ & $0.1794(2)$ & $0.6940(2)$ & $0.0672(7)$ & \\
\hline H6 & 1.305232 & 0.178777 & 0.735651 & $0.081^{*}$ & \\
\hline $\mathrm{C} 7$ & $1.0310(5)$ & $0.2677(2)$ & $0.68807(18)$ & $0.0594(6)$ & \\
\hline $\mathrm{H} 7$ & 1.100928 & 0.326025 & 0.726530 & $0.071^{*}$ & \\
\hline $\mathrm{C} 8$ & $0.8032(4)$ & $0.27050(17)$ & $0.62436(16)$ & $0.0497(5)$ & \\
\hline C9 & $0.7704(5)$ & $0.45374(17)$ & $0.66992(17)$ & $0.0547(6)$ & \\
\hline H9A & 0.711702 & 0.507452 & 0.634775 & $0.066^{*}$ & \\
\hline H9B & 0.957721 & 0.455955 & 0.678226 & $0.066^{*}$ & \\
\hline $\mathrm{C} 10$ & $0.6832(5)$ & $0.47073(19)$ & $0.76690(18)$ & $0.0606(6)$ & \\
\hline $\mathrm{H} 10 \mathrm{~A}$ & 0.498084 & 0.479028 & 0.758694 & $0.073^{*}$ & \\
\hline H10B & 0.717777 & 0.412121 & 0.798120 & $0.073^{*}$ & \\
\hline $\mathrm{C} 11$ & $0.8169(6)$ & $0.5614(2)$ & $0.8298(2)$ & $0.0745(8)$ & \\
\hline $\mathrm{H} 11 \mathrm{~A}$ & 0.798334 & 0.618437 & 0.795459 & $0.089^{*}$ & \\
\hline H11B & 0.999863 & 0.549819 & 0.843410 & $0.089^{*}$ & \\
\hline $\mathrm{C} 12$ & $0.7119(8)$ & 0.5865 & $0.9237(2)$ & $0.0978(11)$ & \\
\hline $\mathrm{H} 12 \mathrm{C}$ & 0.774577 & 0.539337 & 0.966060 & $0.117^{*}$ & $0.396(11)$ \\
\hline H12D & 0.524815 & 0.579172 & 0.911703 & $0.117^{*}$ & $0.396(11)$ \\
\hline $\mathrm{H} 12 \mathrm{~A}$ & 0.555095 & 0.622546 & 0.911469 & $0.117^{*}$ & $0.604(11)$ \\
\hline H12B & 0.663609 & 0.524143 & 0.944183 & $0.117^{*}$ & $0.604(11)$ \\
\hline C13B & $0.791(3)$ & $0.6928(9)$ & $0.9730(12)$ & $0.143(6)$ & $0.396(11)$ \\
\hline H13A & 0.733512 & 0.738151 & 0.928155 & $0.172^{*}$ & $0.396(11)$ \\
\hline H13B & 0.694988 & 0.706615 & 1.026028 & $0.172 *$ & $0.396(11)$ \\
\hline C14B & $1.067(3)$ & $0.7193(11)$ & $1.0101(13)$ & $0.178(8)$ & $0.396(11)$ \\
\hline H14A & 1.144535 & 0.724151 & 0.953271 & $0.214^{*}$ & $0.396(11)$ \\
\hline H14B & 1.131999 & 0.659083 & 1.033095 & $0.214^{*}$ & $0.396(11)$ \\
\hline C15B & $1.203(5)$ & $0.8041(10)$ & $1.0834(14)$ & $0.213(9)$ & $0.396(11)$ \\
\hline
\end{tabular}




\begin{tabular}{|c|c|c|c|c|c|}
\hline $\mathrm{H} 15 \mathrm{~A}$ & 1.115971 & 0.809553 & 1.139137 & $0.255^{*}$ & $0.396(11)$ \\
\hline H15B & 1.377951 & 0.784635 & 1.103084 & $0.255^{*}$ & $0.396(11)$ \\
\hline $\mathrm{C} 13 \mathrm{~A}$ & $0.8860(18)$ & $0.6470(6)$ & $1.0052(4)$ & $0.108(3)$ & $0.604(11)$ \\
\hline $\mathrm{H} 13 \mathrm{C}$ & 0.803887 & 0.653364 & 1.062129 & $0.129 *$ & $0.604(11)$ \\
\hline H13D & 1.046676 & 0.613550 & 1.018024 & $0.129 *$ & $0.604(11)$ \\
\hline C14A & 0.9394 (19) & $0.7469(5)$ & $0.9806(5)$ & $0.109(3)$ & $0.604(11)$ \\
\hline $\mathrm{H} 14 \mathrm{C}$ & 0.778583 & 0.778877 & 0.963392 & $0.130 *$ & $0.604(11)$ \\
\hline H14D & 1.033828 & 0.741313 & 0.926736 & $0.130 *$ & $0.604(11)$ \\
\hline $\mathrm{C} 15 \mathrm{~A}$ & 1.095 & $0.8071(7)$ & $1.0650(5)$ & $0.151(4)$ & $0.604(11)$ \\
\hline $\mathrm{H} 15 \mathrm{C}$ & 0.983493 & 0.820608 & 1.113487 & $0.182 *$ & $0.604(11)$ \\
\hline H15D & 1.229528 & 0.765333 & 1.089666 & $0.182 *$ & $0.604(11)$ \\
\hline $\mathrm{C} 16$ & $1.2192(15)$ & $0.9031(5)$ & $1.0549(5)$ & $0.208(3)$ & \\
\hline $\mathrm{H} 16 \mathrm{C}$ & 1.047455 & 0.927886 & 1.040740 & $0.250 *$ & $0.396(11)$ \\
\hline H16D & 1.302078 & 0.900230 & 0.998269 & $0.250 *$ & $0.396(11)$ \\
\hline H16A & 1.331550 & 0.888114 & 1.006973 & $0.250 *$ & $0.604(11)$ \\
\hline H16B & 1.083193 & 0.942764 & 1.027665 & $0.250^{*}$ & $0.604(11)$ \\
\hline $\mathrm{C} 17$ & $1.3710(9)$ & $0.9691(3)$ & $1.1345(4)$ & $0.1294(16)$ & \\
\hline H17A & 1.353016 & 1.037431 & 1.124155 & $0.194 *$ & \\
\hline H17B & 1.549689 & 0.953042 & 1.138569 & $0.194 *$ & \\
\hline $\mathrm{H} 17 \mathrm{C}$ & 1.309895 & 0.959940 & 1.192911 & $0.194 *$ & \\
\hline $\mathrm{C} 18$ & $0.1379(5)$ & $0.25620(17)$ & $0.41263(17)$ & $0.0517(6)$ & \\
\hline C19 & $0.1175(6)$ & $0.1751(2)$ & 0.33936 (19) & $0.0663(7)$ & \\
\hline H19 & 0.242243 & 0.127165 & 0.340750 & $0.080 *$ & \\
\hline $\mathrm{C} 20$ & $-0.0844(6)$ & $0.1652(2)$ & $0.2653(2)$ & $0.0780(8)$ & \\
\hline $\mathrm{H} 20$ & -0.094842 & 0.110612 & 0.217213 & $0.094 *$ & \\
\hline $\mathrm{C} 21$ & $-0.2709(6)$ & $0.2351(2)$ & $0.2615(2)$ & $0.0749(8)$ & \\
\hline $\mathrm{H} 21$ & -0.408785 & 0.227544 & 0.211843 & $0.090^{*}$ & \\
\hline $\mathrm{C} 22$ & $-0.2513(5)$ & $0.3164(2)$ & $0.3319(2)$ & $0.0680(7)$ & \\
\hline $\mathrm{H} 22$ & -0.375143 & 0.364646 & 0.328908 & $0.082 *$ & \\
\hline $\mathrm{C} 23$ & $-0.0503(5)$ & $0.32754(19)$ & $0.40714(19)$ & $0.0574(6)$ & \\
\hline $\mathrm{H} 23$ & -0.040380 & 0.382880 & 0.454350 & $0.069^{*}$ & \\
\hline
\end{tabular}

Atomic displacement parameters $\left(\AA^{2}\right)$

\begin{tabular}{lllllll}
\hline & $U^{11}$ & $U^{22}$ & $U^{33}$ & $U^{12}$ & $U^{13}$ & $U^{23}$ \\
\hline O1 & $0.0726(11)$ & $0.0453(9)$ & $0.0818(13)$ & $0.0079(8)$ & $0.0004(9)$ & $-0.0005(8)$ \\
N1 & $0.0560(11)$ & $0.0410(10)$ & $0.0511(11)$ & $-0.0013(8)$ & $0.0092(9)$ & $0.0018(8)$ \\
C1 & $0.0567(13)$ & $0.0449(12)$ & $0.0511(13)$ & $0.0017(10)$ & $0.0094(10)$ & $0.0046(10)$ \\
N2 & $0.0590(11)$ & $0.0431(10)$ & $0.0556(11)$ & $0.0006(8)$ & $0.0100(9)$ & $0.0046(8)$ \\
C2 & $0.0544(12)$ & $0.0427(11)$ & $0.0506(12)$ & $-0.0014(9)$ & $0.0124(10)$ & $0.0058(9)$ \\
C3 & $0.0596(13)$ & $0.0458(12)$ & $0.0503(13)$ & $0.0015(10)$ & $0.0110(10)$ & $0.0071(10)$ \\
C4 & $0.0740(16)$ & $0.0466(13)$ & $0.0672(16)$ & $0.0068(12)$ & $0.0115(13)$ & $0.0079(11)$ \\
C5 & $0.0749(17)$ & $0.0602(16)$ & $0.0772(18)$ & $0.0160(13)$ & $0.0096(14)$ & $0.0203(14)$ \\
C6 & $0.0635(15)$ & $0.0762(18)$ & $0.0627(16)$ & $0.0087(13)$ & $0.0034(12)$ & $0.0179(13)$ \\
C7 & $0.0627(14)$ & $0.0593(15)$ & $0.0545(14)$ & $0.0002(11)$ & $0.0060(11)$ & $0.0058(11)$ \\
C8 & $0.0567(13)$ & $0.0466(12)$ & $0.0480(12)$ & $0.0014(10)$ & $0.0143(10)$ & $0.0083(9)$ \\
C9 & $0.0591(13)$ & $0.0446(12)$ & $0.0574(14)$ & $-0.0047(10)$ & $0.0057(11)$ & $0.0014(10)$ \\
C10 & $0.0643(15)$ & $0.0564(14)$ & $0.0583(15)$ & $-0.0017(11)$ & $0.0083(12)$ & $0.0000(11)$
\end{tabular}




$\begin{array}{lllllll}\text { C11 } & 0.0707(17) & 0.0774(18) & 0.0667(16) & -0.0048(14) & 0.0051(13) & -0.0135(14) \\ \text { C12 } & 0.110(3) & 0.101(2) & 0.075(2) & 0.001(2) & 0.0198(18) & -0.0184(18) \\ \text { C13B } & 0.185(12) & 0.124(9) & 0.100(11) & -0.017(9) & 0.017(8) & -0.049(8) \\ \text { C14B } & 0.234(15) & 0.157(11) & 0.116(11) & -0.097(12) & -0.048(11) & 0.019(9) \\ \text { C15B } & 0.204(19) & 0.152(8) & 0.249(18) & -0.094(11) & 0.037(13) & -0.073(8) \\ \text { C13A } & 0.143(6) & 0.120(5) & 0.054(3) & -0.002(4) & 0.009(3) & -0.004(3) \\ \text { C14A } & 0.128(7) & 0.119(5) & 0.067(4) & -0.024(4) & 0.009(4) & -0.015(3) \\ \text { C15A } & 0.192(11) & 0.174(7) & 0.070(4) & -0.062(6) & 0.028(5) & -0.047(4) \\ \text { C16 } & 0.213(7) & 0.174(5) & 0.206(7) & -0.081(5) & 0.012(5) & -0.051(5) \\ \text { C17 } & 0.123(3) & 0.099(3) & 0.146(4) & -0.008(2) & -0.013(3) & -0.015(3) \\ \text { C18 } & 0.0560(13) & 0.0455(12) & 0.0544(13) & -0.0054(10) & 0.0102(10) & 0.0089(10) \\ \text { C19 } & 0.0733(17) & 0.0538(14) & 0.0655(16) & 0.0023(12) & -0.0016(13) & -0.0008(12) \\ \text { C20 } & 0.086(2) & 0.0679(18) & 0.0694(18) & -0.0039(15) & -0.0073(15) & -0.0054(14) \\ \text { C21 } & 0.0684(17) & 0.081(2) & 0.0702(18) & -0.0068(15) & -0.0100(14) & 0.0153(15) \\ \text { C22 } & 0.0617(15) & 0.0711(17) & 0.0718(17) & 0.0044(13) & 0.0051(13) & 0.0174(14) \\ \text { C23 } & 0.0570(13) & 0.0554(13) & 0.0616(14) & -0.0001(11) & 0.0122(11) & 0.0114(11)\end{array}$

Geometric parameters $\left(\stackrel{A}{A}{ }^{\circ}\right)$

\begin{tabular}{|c|c|c|c|}
\hline $\mathrm{O} 1-\mathrm{C} 1$ & $1.221(3)$ & $\mathrm{C} 7-\mathrm{C} 8$ & $1.397(3)$ \\
\hline $\mathrm{C} 13 \mathrm{Bb}-\mathrm{H} 13 \mathrm{~A}$ & 0.9700 & C9-H9A & 0.9700 \\
\hline $\mathrm{C} 13 \mathrm{Bb}-\mathrm{H} 13 \mathrm{~B}$ & 0.9700 & C9-H9B & 0.9700 \\
\hline $\mathrm{C} 13 \mathrm{Bb}-\mathrm{C} 14 \mathrm{~B}$ & $1.482(10)$ & $\mathrm{C} 9-\mathrm{C} 10$ & $1.512(3)$ \\
\hline $\mathrm{C} 14 \mathrm{Bb}-\mathrm{H} 14 \mathrm{~A}$ & 0.9700 & $\mathrm{C} 10-\mathrm{H} 10 \mathrm{~A}$ & 0.9700 \\
\hline $\mathrm{C} 14 \mathrm{Bb}-\mathrm{H} 14 \mathrm{~B}$ & 0.9700 & $\mathrm{C} 10-\mathrm{H} 10 \mathrm{~B}$ & 0.9700 \\
\hline $\mathrm{C} 14 \mathrm{Bb}-\mathrm{C} 15 \mathrm{~B}$ & $1.527(9)$ & $\mathrm{C} 10-\mathrm{C} 11$ & $1.507(4)$ \\
\hline $\mathrm{C} 15 \mathrm{Bb}-\mathrm{H} 15 \mathrm{~A}$ & 0.9700 & $\mathrm{C} 11-\mathrm{H} 11 \mathrm{~A}$ & 0.9700 \\
\hline $\mathrm{C} 15 \mathrm{Bb}-\mathrm{H} 15 \mathrm{~B}$ & 0.9700 & C11-H11B & 0.9700 \\
\hline $\mathrm{C} 13 \mathrm{Aa}-\mathrm{H} 13 \mathrm{C}$ & 0.9700 & $\mathrm{C} 11-\mathrm{C} 12$ & $1.523(4)$ \\
\hline $\mathrm{C} 13 \mathrm{Aa}-\mathrm{H} 13 \mathrm{D}$ & 0.9700 & $\mathrm{C} 12-\mathrm{H} 12 \mathrm{C}$ & 0.9700 \\
\hline $\mathrm{C} 13 \mathrm{Aa}-\mathrm{C} 14 \mathrm{~A}$ & $1.472(8)$ & $\mathrm{C} 12-\mathrm{H} 12 \mathrm{D}$ & 0.9700 \\
\hline $\mathrm{C} 14 \mathrm{Aa}-\mathrm{H} 14 \mathrm{C}$ & 0.9700 & $\mathrm{C} 12-\mathrm{H} 12 \mathrm{~A}$ & 0.9700 \\
\hline C14Aa-H14D & 0.9700 & $\mathrm{C} 12-\mathrm{H} 12 \mathrm{~B}$ & 0.9700 \\
\hline $\mathrm{C} 14 \mathrm{Aa}-\mathrm{C} 15 \mathrm{~A}$ & $1.481(7)$ & $\mathrm{C} 12-\mathrm{C} 13 \mathrm{~B}$ & $1.528(9)$ \\
\hline $\mathrm{C} 15 \mathrm{Aa}-\mathrm{H} 15 \mathrm{C}$ & 0.9700 & $\mathrm{C} 12-\mathrm{C} 13 \mathrm{~A}$ & $1.500(6)$ \\
\hline $\mathrm{C} 15 \mathrm{Aa}-\mathrm{H} 15 \mathrm{D}$ & 0.9700 & $\mathrm{C} 16-\mathrm{H} 16 \mathrm{C}$ & 0.9700 \\
\hline $\mathrm{C} 15 \mathrm{Bb}-\mathrm{C} 16$ & $1.456(10)$ & $\mathrm{C} 16-\mathrm{H} 16 \mathrm{D}$ & 0.9700 \\
\hline $\mathrm{C} 15 \mathrm{Aa}-\mathrm{C} 16$ & $1.473(5)$ & $\mathrm{C} 16-\mathrm{H} 16 \mathrm{~A}$ & 0.9700 \\
\hline $\mathrm{N} 1-\mathrm{C} 1$ & $1.379(3)$ & $\mathrm{C} 16-\mathrm{H} 16 \mathrm{~B}$ & 0.9700 \\
\hline $\mathrm{N} 1-\mathrm{C} 8$ & $1.387(3)$ & $\mathrm{C} 16-\mathrm{C} 17$ & $1.466(7)$ \\
\hline $\mathrm{N} 1-\mathrm{C} 9$ & $1.474(3)$ & $\mathrm{C} 17-\mathrm{H} 17 \mathrm{~A}$ & 0.9600 \\
\hline $\mathrm{C} 1-\mathrm{C} 2$ & $1.496(3)$ & $\mathrm{C} 17-\mathrm{H} 17 \mathrm{~B}$ & 0.9600 \\
\hline $\mathrm{N} 2-\mathrm{C} 2$ & $1.296(3)$ & $\mathrm{C} 17-\mathrm{H} 17 \mathrm{C}$ & 0.9600 \\
\hline $\mathrm{N} 2-\mathrm{C} 3$ & $1.384(3)$ & $\mathrm{C} 18-\mathrm{C} 19$ & $1.397(3)$ \\
\hline $\mathrm{C} 2-\mathrm{C} 18$ & $1.481(3)$ & $\mathrm{C} 18-\mathrm{C} 23$ & $1.395(3)$ \\
\hline $\mathrm{C} 3-\mathrm{C} 4$ & $1.399(3)$ & C19-H19 & 0.9300 \\
\hline $\mathrm{C} 3-\mathrm{C} 8$ & $1.410(3)$ & $\mathrm{C} 19-\mathrm{C} 20$ & $1.375(4)$ \\
\hline $\mathrm{C} 4-\mathrm{H} 4$ & 0.9300 & $\mathrm{C} 20-\mathrm{H} 20$ & 0.9300 \\
\hline
\end{tabular}




\begin{tabular}{|c|c|c|c|}
\hline $\mathrm{C} 4-\mathrm{C} 5$ & $1.373(4)$ & $\mathrm{C} 20-\mathrm{C} 21$ & $1.374(4)$ \\
\hline $\mathrm{C} 5-\mathrm{H} 5$ & 0.9300 & $\mathrm{C} 21-\mathrm{H} 21$ & 0.9300 \\
\hline $\mathrm{C} 5-\mathrm{C} 6$ & $1.385(4)$ & $\mathrm{C} 21-\mathrm{C} 22$ & $1.374(4)$ \\
\hline $\mathrm{C} 6-\mathrm{H} 6$ & 0.9300 & $\mathrm{C} 22-\mathrm{H} 22$ & 0.9300 \\
\hline $\mathrm{C} 6-\mathrm{C} 7$ & $1.369(4)$ & $\mathrm{C} 22-\mathrm{C} 23$ & $1.382(4)$ \\
\hline $\mathrm{C} 7-\mathrm{H} 7$ & 0.9300 & $\mathrm{C} 23-\mathrm{H} 23$ & 0.9300 \\
\hline $\mathrm{C} 1-\mathrm{N} 1-\mathrm{C} 8$ & $122.74(19)$ & $\mathrm{C} 15 \mathrm{Bb}-\mathrm{C} 16-\mathrm{C} 17$ & $107.4(8)$ \\
\hline $\mathrm{C} 14 \mathrm{Aa}-\mathrm{C} 13 \mathrm{Aa}-\mathrm{C} 12$ & $109.4(6)$ & $\mathrm{N} 1-\mathrm{C} 8-\mathrm{C} 7$ & $123.5(2)$ \\
\hline $\mathrm{C} 14 \mathrm{Bb}-\mathrm{C} 13 \mathrm{Bb}-\mathrm{C} 12$ & $119.6(10)$ & $\mathrm{C} 7-\mathrm{C} 8-\mathrm{C} 3$ & $118.9(2)$ \\
\hline $\mathrm{C} 14 \mathrm{Bb}-\mathrm{C} 13 \mathrm{Bb}-\mathrm{H} 13 \mathrm{~A}$ & 107.4 & $\mathrm{~N} 1-\mathrm{C} 9-\mathrm{H} 9 \mathrm{~A}$ & 109.1 \\
\hline $\mathrm{H} 13 \mathrm{Ab}-\mathrm{C} 13 \mathrm{Bb}-\mathrm{H} 13 \mathrm{~B}$ & 106.9 & $\mathrm{~N} 1-\mathrm{C} 9-\mathrm{H} 9 \mathrm{~B}$ & 109.1 \\
\hline $\mathrm{C} 14 \mathrm{Bb}-\mathrm{C} 13 \mathrm{Bb}-\mathrm{H} 13 \mathrm{~B}$ & 107.4 & $\mathrm{~N} 1-\mathrm{C} 9-\mathrm{C} 10$ & $112.61(19)$ \\
\hline $\mathrm{C} 13 \mathrm{Bb}-\mathrm{C} 14 \mathrm{Bb}-\mathrm{H} 14 \mathrm{~A}$ & 104.0 & $\mathrm{H} 9 \mathrm{~A}-\mathrm{C} 9-\mathrm{H} 9 \mathrm{~B}$ & 107.8 \\
\hline $\mathrm{C} 1-\mathrm{N} 1-\mathrm{C} 9$ & $116.64(19)$ & $\mathrm{C} 10-\mathrm{C} 9-\mathrm{H} 9 \mathrm{~A}$ & 109.1 \\
\hline $\mathrm{C} 8-\mathrm{N} 1-\mathrm{C} 9$ & $120.60(19)$ & $\mathrm{C} 10-\mathrm{C} 9-\mathrm{H} 9 \mathrm{~B}$ & 109.1 \\
\hline $\mathrm{O} 1-\mathrm{C} 1-\mathrm{N} 1$ & $120.6(2)$ & $\mathrm{C} 9-\mathrm{C} 10-\mathrm{H} 10 \mathrm{~A}$ & 109.1 \\
\hline $\mathrm{O} 1-\mathrm{C} 1-\mathrm{C} 2$ & $124.1(2)$ & $\mathrm{C} 9-\mathrm{C} 10-\mathrm{H} 10 \mathrm{~B}$ & 109.1 \\
\hline $\mathrm{C} 15 \mathrm{Bb}-\mathrm{C} 14 \mathrm{Bb}-\mathrm{H} 14 \mathrm{~A}$ & 104.0 & $\mathrm{H} 10 \mathrm{~A}-\mathrm{C} 10-\mathrm{H} 10 \mathrm{~B}$ & 107.8 \\
\hline $\mathrm{H} 14 \mathrm{Ab}-\mathrm{C} 14 \mathrm{Bb}-\mathrm{H} 14 \mathrm{~B}$ & 105.4 & $\mathrm{C} 11-\mathrm{C} 10-\mathrm{C} 9$ & $112.5(2)$ \\
\hline $\mathrm{C} 13 \mathrm{Bb}-\mathrm{C} 14 \mathrm{Bb}-\mathrm{H} 14 \mathrm{~B}$ & 104.0 & $\mathrm{C} 11-\mathrm{C} 10-\mathrm{H} 10 \mathrm{~A}$ & 109.1 \\
\hline $\mathrm{C} 15 \mathrm{Bb}-\mathrm{C} 14 \mathrm{Bb}-\mathrm{H} 14 \mathrm{~B}$ & 104.0 & $\mathrm{C} 11-\mathrm{C} 10-\mathrm{H} 10 \mathrm{~B}$ & 109.1 \\
\hline $\mathrm{C} 13 \mathrm{Bb}-\mathrm{C} 14 \mathrm{Bb}-\mathrm{C} 15 \mathrm{~B}$ & $133.0(17)$ & $\mathrm{C} 10-\mathrm{C} 11-\mathrm{H} 11 \mathrm{~A}$ & 108.9 \\
\hline $\mathrm{C} 14 \mathrm{Bb}-\mathrm{C} 15 \mathrm{Bb}-\mathrm{H} 15 \mathrm{~A}$ & 107.9 & $\mathrm{C} 10-\mathrm{C} 11-\mathrm{H} 11 \mathrm{~B}$ & 108.9 \\
\hline $\mathrm{C} 14 \mathrm{Bb}-\mathrm{C} 15 \mathrm{Bb}-\mathrm{H} 15 \mathrm{~B}$ & 107.9 & $\mathrm{C} 10-\mathrm{C} 11-\mathrm{C} 12$ & $113.5(3)$ \\
\hline $\mathrm{H} 15 \mathrm{Ab}-\mathrm{C} 15 \mathrm{Bb}-\mathrm{H} 15 \mathrm{~B}$ & 107.2 & $\mathrm{H} 11 \mathrm{~A}-\mathrm{C} 11-\mathrm{H} 11 \mathrm{~B}$ & 107.7 \\
\hline $\mathrm{N} 1-\mathrm{C} 1-\mathrm{C} 2$ & $115.22(19)$ & $\mathrm{C} 12-\mathrm{C} 11-\mathrm{H} 11 \mathrm{~A}$ & 108.9 \\
\hline $\mathrm{C} 2-\mathrm{N} 2-\mathrm{C} 3$ & $120.3(2)$ & $\mathrm{C} 12-\mathrm{C} 11-\mathrm{H} 11 \mathrm{~B}$ & 108.9 \\
\hline $\mathrm{N} 2-\mathrm{C} 2-\mathrm{C} 1$ & $122.0(2)$ & $\mathrm{C} 11-\mathrm{C} 12-\mathrm{H} 12 \mathrm{C}$ & 109.1 \\
\hline $\mathrm{N} 2-\mathrm{C} 2-\mathrm{C} 18$ & $117.6(2)$ & $\mathrm{C} 11-\mathrm{C} 12-\mathrm{H} 12 \mathrm{D}$ & 109.1 \\
\hline $\mathrm{C} 18-\mathrm{C} 2-\mathrm{C} 1$ & $120.4(2)$ & $\mathrm{C} 11-\mathrm{C} 12-\mathrm{H} 12 \mathrm{~A}$ & 107.9 \\
\hline $\mathrm{N} 2-\mathrm{C} 3-\mathrm{C} 4$ & $118.7(2)$ & $\mathrm{C} 11-\mathrm{C} 12-\mathrm{H} 12 \mathrm{~B}$ & 107.9 \\
\hline $\mathrm{N} 2-\mathrm{C} 3-\mathrm{C} 8$ & $121.6(2)$ & $\mathrm{C} 11-\mathrm{C} 12-\mathrm{C} 13 \mathrm{~B}$ & $112.6(8)$ \\
\hline $\mathrm{C} 4-\mathrm{C} 3-\mathrm{C} 8$ & $119.7(2)$ & $\mathrm{C} 17-\mathrm{C} 16-\mathrm{C} 15 \mathrm{~A}$ & $123.6(7)$ \\
\hline $\mathrm{C} 3-\mathrm{C} 4-\mathrm{H} 4$ & 119.9 & $\mathrm{C} 17-\mathrm{C} 16-\mathrm{H} 16 \mathrm{C}$ & 110.2 \\
\hline $\mathrm{C} 5-\mathrm{C} 4-\mathrm{C} 3$ & $120.2(2)$ & $\mathrm{C} 12-\mathrm{C} 13 \mathrm{Bb}-\mathrm{H} 13 \mathrm{~A}$ & 107.4 \\
\hline $\mathrm{C} 5-\mathrm{C} 4-\mathrm{H} 4$ & 119.9 & $\mathrm{C} 12-\mathrm{C} 13 \mathrm{Bb}-\mathrm{H} 13 \mathrm{~B}$ & 107.4 \\
\hline $\mathrm{C} 14 \mathrm{Aa}-\mathrm{C} 13 \mathrm{Aa}-\mathrm{H} 13 \mathrm{C}$ & 109.8 & $\mathrm{C} 12-\mathrm{C} 13 \mathrm{Aa}-\mathrm{H} 13 \mathrm{C}$ & 109.8 \\
\hline $\mathrm{C} 14 \mathrm{Aa}-\mathrm{C} 13 \mathrm{Aa}-\mathrm{H} 13 \mathrm{D}$ & 109.8 & $\mathrm{C} 12-\mathrm{C} 13 \mathrm{Aa}-\mathrm{H} 13 \mathrm{D}$ & 109.8 \\
\hline $\mathrm{H} 13 \mathrm{Ca}-\mathrm{C} 13 \mathrm{Aa}-\mathrm{H} 13 \mathrm{D}$ & 108.2 & $\mathrm{C} 16-\mathrm{C} 15 \mathrm{Bb}-\mathrm{C} 14 \mathrm{~B}$ & $117.8(12)$ \\
\hline $\mathrm{C} 13 \mathrm{Aa}-\mathrm{C} 14 \mathrm{Aa}-\mathrm{H} 14 \mathrm{C}$ & 110.1 & $\mathrm{C} 17-\mathrm{C} 16-\mathrm{H} 16 \mathrm{D}$ & 110.2 \\
\hline $\mathrm{C} 15 \mathrm{Aa}-\mathrm{C} 14 \mathrm{Aa}-\mathrm{H} 14 \mathrm{C}$ & 110.1 & $\mathrm{C} 17-\mathrm{C} 16-\mathrm{H} 16 \mathrm{~A}$ & 106.4 \\
\hline $\mathrm{C} 15 \mathrm{Aa}-\mathrm{C} 14 \mathrm{Aa}-\mathrm{H} 14 \mathrm{D}$ & 110.1 & $\mathrm{C} 17-\mathrm{C} 16-\mathrm{H} 16 \mathrm{~B}$ & 106.4 \\
\hline $\mathrm{H} 14 \mathrm{Ca}-\mathrm{C} 14 \mathrm{Aa}-\mathrm{H} 14 \mathrm{D}$ & 108.4 & $\mathrm{C} 16-\mathrm{C} 17-\mathrm{H} 17 \mathrm{~A}$ & 109.5 \\
\hline $\mathrm{C} 13 \mathrm{Aa}-\mathrm{C} 14 \mathrm{Aa}-\mathrm{H} 14 \mathrm{D}$ & 110.1 & $\mathrm{C} 16-\mathrm{C} 17-\mathrm{H} 17 \mathrm{~B}$ & 109.5 \\
\hline $\mathrm{C} 13 \mathrm{Aa}-\mathrm{C} 14 \mathrm{Aa}-\mathrm{C} 15 \mathrm{~A}$ & $108.0(6)$ & $\mathrm{C} 16-\mathrm{C} 17-\mathrm{H} 17 \mathrm{C}$ & 109.5 \\
\hline $\mathrm{C} 14 \mathrm{Aa}-\mathrm{C} 15 \mathrm{Aa}-\mathrm{H} 15 \mathrm{C}$ & 107.4 & $\mathrm{H} 17 \mathrm{~A}-\mathrm{C} 17-\mathrm{H} 17 \mathrm{~B}$ & 109.5 \\
\hline $\mathrm{C} 14 \mathrm{Aa}-\mathrm{C} 15 \mathrm{Aa}-\mathrm{H} 15 \mathrm{D}$ & 107.4 & $\mathrm{H} 17 \mathrm{~A}-\mathrm{C} 17-\mathrm{H} 17 \mathrm{C}$ & 109.5 \\
\hline
\end{tabular}




\begin{tabular}{|c|c|c|c|}
\hline $\mathrm{C} 4-\mathrm{C} 5-\mathrm{H} 5$ & 120.1 & $\mathrm{H} 17 \mathrm{~B}-\mathrm{C} 17-\mathrm{H} 17 \mathrm{C}$ & 109.5 \\
\hline $\mathrm{C} 4-\mathrm{C} 5-\mathrm{C} 6$ & $119.9(2)$ & $\mathrm{C} 19-\mathrm{C} 18-\mathrm{C} 2$ & $117.8(2)$ \\
\hline $\mathrm{C} 6-\mathrm{C} 5-\mathrm{H} 5$ & 120.1 & $\mathrm{C} 23-\mathrm{C} 18-\mathrm{C} 2$ & $124.4(2)$ \\
\hline $\mathrm{C} 5-\mathrm{C} 6-\mathrm{H} 6$ & 119.4 & $\mathrm{C} 23-\mathrm{C} 18-\mathrm{C} 19$ & $117.9(2)$ \\
\hline $\mathrm{C} 7-\mathrm{C} 6-\mathrm{C} 5$ & $121.2(3)$ & $\mathrm{C} 18-\mathrm{C} 19-\mathrm{H} 19$ & 119.5 \\
\hline $\mathrm{C} 7-\mathrm{C} 6-\mathrm{H} 6$ & 119.4 & $\mathrm{C} 20-\mathrm{C} 19-\mathrm{C} 18$ & $120.9(3)$ \\
\hline $\mathrm{C} 6-\mathrm{C} 7-\mathrm{H} 7$ & 119.9 & $\mathrm{C} 20-\mathrm{C} 19-\mathrm{H} 19$ & 119.5 \\
\hline $\mathrm{C} 6-\mathrm{C} 7-\mathrm{C} 8$ & $120.2(2)$ & $\mathrm{C} 19-\mathrm{C} 20-\mathrm{H} 20$ & 119.7 \\
\hline $\mathrm{C} 8-\mathrm{C} 7-\mathrm{H} 7$ & 119.9 & $\mathrm{C} 21-\mathrm{C} 20-\mathrm{C} 19$ & $120.6(3)$ \\
\hline $\mathrm{N} 1-\mathrm{C} 8-\mathrm{C} 3$ & $117.6(2)$ & $\mathrm{C} 21-\mathrm{C} 20-\mathrm{H} 20$ & 119.7 \\
\hline $\mathrm{H} 15 \mathrm{Ca}-\mathrm{C} 15 \mathrm{Aa}-\mathrm{H} 15 \mathrm{D}$ & 107.0 & $\mathrm{C} 20-\mathrm{C} 21-\mathrm{H} 21$ & 120.4 \\
\hline $\mathrm{C} 13 \mathrm{Aa}-\mathrm{C} 12-\mathrm{C} 11$ & $117.4(5)$ & $\mathrm{C} 22-\mathrm{C} 21-\mathrm{C} 20$ & $119.3(3)$ \\
\hline $\mathrm{C} 13 \mathrm{Bb}-\mathrm{C} 12-\mathrm{H} 12 \mathrm{C}$ & 109.1 & $\mathrm{C} 22-\mathrm{C} 21-\mathrm{H} 21$ & 120.4 \\
\hline $\mathrm{H} 12 \mathrm{Cb}-\mathrm{C} 12-\mathrm{H} 12 \mathrm{D}$ & 107.8 & $\mathrm{C} 21-\mathrm{C} 22-\mathrm{H} 22$ & 119.5 \\
\hline $\mathrm{C} 13 \mathrm{Bb}-\mathrm{C} 12-\mathrm{H} 12 \mathrm{D}$ & 109.1 & $\mathrm{C} 21-\mathrm{C} 22-\mathrm{C} 23$ & $121.0(3)$ \\
\hline $\mathrm{C} 13 \mathrm{Aa}-\mathrm{C} 12-\mathrm{H} 12 \mathrm{~A}$ & 107.9 & $\mathrm{C} 23-\mathrm{C} 22-\mathrm{H} 22$ & 119.5 \\
\hline $\mathrm{C} 13 \mathrm{Aa}-\mathrm{C} 12-\mathrm{H} 12 \mathrm{~B}$ & 107.9 & $\mathrm{C} 18-\mathrm{C} 23-\mathrm{H} 23$ & 119.8 \\
\hline $\mathrm{H} 12 \mathrm{Aa}-\mathrm{C} 12-\mathrm{H} 12 \mathrm{~B}$ & 107.2 & $\mathrm{C} 22-\mathrm{C} 23-\mathrm{C} 18$ & $120.3(3)$ \\
\hline $\mathrm{C} 15 \mathrm{Bb}-\mathrm{C} 16-\mathrm{H} 16 \mathrm{C}$ & 110.2 & $\mathrm{C} 22-\mathrm{C} 23-\mathrm{H} 23$ & 119.8 \\
\hline $\mathrm{C} 15 \mathrm{Bb}-\mathrm{C} 16-\mathrm{H} 16 \mathrm{D}$ & 110.2 & $\mathrm{C} 16-\mathrm{C} 15 \mathrm{Bb}-\mathrm{H} 15 \mathrm{~A}$ & 107.9 \\
\hline $\mathrm{H} 16 \mathrm{Cb}-\mathrm{C} 16-\mathrm{H} 16 \mathrm{D}$ & 108.5 & $\mathrm{C} 16-\mathrm{C} 15 \mathrm{Bb}-\mathrm{H} 15 \mathrm{~B}$ & 107.9 \\
\hline $\mathrm{C} 15 \mathrm{Aa}-\mathrm{C} 16-\mathrm{H} 16 \mathrm{~A}$ & 106.4 & $\mathrm{C} 16-\mathrm{C} 15 \mathrm{Aa}-\mathrm{C} 14 \mathrm{~A}$ & $119.5(7)$ \\
\hline $\mathrm{H} 16 \mathrm{Aa}-\mathrm{C} 16-\mathrm{H} 16 \mathrm{~B}$ & 106.5 & $\mathrm{C} 16-\mathrm{C} 15 \mathrm{Aa}-\mathrm{H} 15 \mathrm{C}$ & 107.4 \\
\hline $\mathrm{C} 15 \mathrm{Aa}-\mathrm{C} 16-\mathrm{H} 16 \mathrm{~B}$ & 106.4 & $\mathrm{C} 16-\mathrm{C} 15 \mathrm{Aa}-\mathrm{H} 15 \mathrm{D}$ & 107.4 \\
\hline $\mathrm{C} 13 \mathrm{Aa}-\mathrm{C} 14 \mathrm{Aa}-\mathrm{C} 15 \mathrm{Aa}-\mathrm{C} 16$ & $168.0(12)$ & $\mathrm{C} 4-\mathrm{C} 3-\mathrm{C} 8-\mathrm{C} 7$ & $0.7(4)$ \\
\hline $\mathrm{C} 13 \mathrm{Bb}-\mathrm{C} 14 \mathrm{Bb}-\mathrm{C} 15 \mathrm{Bb}-\mathrm{C} 16$ & $-76(3)$ & $\mathrm{C} 4-\mathrm{C} 5-\mathrm{C} 6-\mathrm{C} 7$ & $1.1(4)$ \\
\hline $\mathrm{C} 14 \mathrm{Bb}-\mathrm{C} 15 \mathrm{Bb}-\mathrm{C} 16-\mathrm{C} 17$ & $-176.5(18)$ & $\mathrm{C} 5-\mathrm{C} 6-\mathrm{C} 7-\mathrm{C} 8$ & $-1.4(4)$ \\
\hline $\mathrm{C} 14 \mathrm{Aa}-\mathrm{C} 15 \mathrm{Aa}-\mathrm{C} 16-\mathrm{C} 17$ & $178.4(9)$ & $\mathrm{C} 6-\mathrm{C} 7-\mathrm{C} 8-\mathrm{N} 1$ & $-179.5(2)$ \\
\hline $\mathrm{O} 1-\mathrm{C} 1-\mathrm{C} 2-\mathrm{N} 2$ & $-172.5(2)$ & $\mathrm{C} 6-\mathrm{C} 7-\mathrm{C} 8-\mathrm{C} 3$ & $0.5(4)$ \\
\hline $\mathrm{O} 1-\mathrm{C} 1-\mathrm{C} 2-\mathrm{C} 18$ & $9.4(4)$ & $\mathrm{C} 8-\mathrm{N} 1-\mathrm{C} 1-\mathrm{O} 1$ & $172.5(2)$ \\
\hline $\mathrm{N} 1-\mathrm{C} 1-\mathrm{C} 2-\mathrm{N} 2$ & $7.6(3)$ & $\mathrm{C} 8-\mathrm{N} 1-\mathrm{C} 1-\mathrm{C} 2$ & $-7.6(3)$ \\
\hline $\mathrm{N} 1-\mathrm{C} 1-\mathrm{C} 2-\mathrm{C} 18$ & $-170.52(19)$ & $\mathrm{C} 8-\mathrm{N} 1-\mathrm{C} 9-\mathrm{C} 10$ & $-86.1(3)$ \\
\hline $\mathrm{N} 1-\mathrm{C} 9-\mathrm{C} 10-\mathrm{C} 11$ & $171.1(2)$ & $\mathrm{C} 8-\mathrm{C} 3-\mathrm{C} 4-\mathrm{C} 5$ & $-1.1(4)$ \\
\hline $\mathrm{C} 1-\mathrm{N} 1-\mathrm{C} 8-\mathrm{C} 3$ & $2.8(3)$ & $\mathrm{C} 9-\mathrm{N} 1-\mathrm{C} 1-\mathrm{O} 1$ & $-5.8(3)$ \\
\hline $\mathrm{C} 1-\mathrm{N} 1-\mathrm{C} 8-\mathrm{C} 7$ & $-177.2(2)$ & $\mathrm{C} 9-\mathrm{N} 1-\mathrm{C} 1-\mathrm{C} 2$ & $174.13(19)$ \\
\hline $\mathrm{C} 1-\mathrm{N} 1-\mathrm{C} 9-\mathrm{C} 10$ & $92.3(3)$ & $\mathrm{C} 9-\mathrm{N} 1-\mathrm{C} 8-\mathrm{C} 3$ & $-179.0(2)$ \\
\hline $\mathrm{C} 1-\mathrm{C} 2-\mathrm{C} 18-\mathrm{C} 19$ & $160.9(2)$ & $\mathrm{C} 9-\mathrm{N} 1-\mathrm{C} 8-\mathrm{C} 7$ & $1.0(3)$ \\
\hline $\mathrm{C} 1-\mathrm{C} 2-\mathrm{C} 18-\mathrm{C} 23$ & $-18.6(3)$ & $\mathrm{C} 9-\mathrm{C} 10-\mathrm{C} 11-\mathrm{C} 12$ & $173.8(3)$ \\
\hline $\mathrm{N} 2-\mathrm{C} 2-\mathrm{C} 18-\mathrm{C} 19$ & $-17.3(3)$ & $\mathrm{C} 10-\mathrm{C} 11-\mathrm{C} 12-\mathrm{C} 13 \mathrm{Aa}$ & $157.2(5)$ \\
\hline $\mathrm{N} 2-\mathrm{C} 2-\mathrm{C} 18-\mathrm{C} 23$ & $163.2(2)$ & $\mathrm{C} 10-\mathrm{C} 11-\mathrm{C} 12-\mathrm{C} 13 \mathrm{Bb}$ & $-163.5(7)$ \\
\hline $\mathrm{N} 2-\mathrm{C} 3-\mathrm{C} 4-\mathrm{C} 5$ & $176.9(2)$ & $\mathrm{C} 11-\mathrm{C} 12-\mathrm{C} 13 \mathrm{Bb}-\mathrm{C} 14 \mathrm{Bb}$ & $-66.5(19)$ \\
\hline $\mathrm{N} 2-\mathrm{C} 3-\mathrm{C} 8-\mathrm{N} 1$ & $2.9(3)$ & $\mathrm{C} 11-\mathrm{C} 12-\mathrm{C} 13 \mathrm{Aa}-\mathrm{C} 14 \mathrm{Aa}$ & $64.2(9)$ \\
\hline $\mathrm{N} 2-\mathrm{C} 3-\mathrm{C} 8-\mathrm{C} 7$ & $-177.2(2)$ & $\mathrm{C} 12-\mathrm{C} 13 \mathrm{Aa}-\mathrm{C} 14 \mathrm{Aa}-\mathrm{C} 15 \mathrm{Aa}$ & $175.8(8)$ \\
\hline $\mathrm{C} 2-\mathrm{N} 2-\mathrm{C} 3-\mathrm{C} 4$ & $179.2(2)$ & $\mathrm{C} 12-\mathrm{C} 13 \mathrm{Bb}-\mathrm{C} 14 \mathrm{Bb}-\mathrm{C} 15 \mathrm{Bb}$ & $-160.7(14)$ \\
\hline $\mathrm{C} 2-\mathrm{N} 2-\mathrm{C} 3-\mathrm{C} 8$ & $-2.8(3)$ & $\mathrm{C} 18-\mathrm{C} 19-\mathrm{C} 20-\mathrm{C} 21$ & $0.1(5)$ \\
\hline $\mathrm{C} 2-\mathrm{C} 18-\mathrm{C} 19-\mathrm{C} 20$ & $179.3(3)$ & $\mathrm{C} 19-\mathrm{C} 18-\mathrm{C} 23-\mathrm{C} 22$ & $1.0(4)$ \\
\hline $\mathrm{C} 2-\mathrm{C} 18-\mathrm{C} 23-\mathrm{C} 22$ & $-179.5(2)$ & $\mathrm{C} 19-\mathrm{C} 20-\mathrm{C} 21-\mathrm{C} 22$ & $1.1(5)$ \\
\hline
\end{tabular}




\begin{tabular}{llll}
$\mathrm{C} 3-\mathrm{N} 2-\mathrm{C} 2-\mathrm{C} 1$ & $-2.5(3)$ & $\mathrm{C} 20-\mathrm{C} 21-\mathrm{C} 22-\mathrm{C} 23$ & $-1.3(5)$ \\
$\mathrm{C} 3-\mathrm{N} 2-\mathrm{C} 2-\mathrm{C} 18$ & $175.63(19)$ & $\mathrm{C} 21-\mathrm{C} 22-\mathrm{C} 23-\mathrm{C} 18$ & $0.3(4)$ \\
$\mathrm{C} 3-\mathrm{C} 4-\mathrm{C} 5-\mathrm{C} 6$ & $0.2(4)$ & $\mathrm{C} 23-\mathrm{C} 18-\mathrm{C} 19-\mathrm{C} 20$ & $-1.2(4)$ \\
$\mathrm{C} 4-\mathrm{C} 3-\mathrm{C} 8-\mathrm{N} 1$ & $-179.3(2)$ & & \\
\hline
\end{tabular}

Hydrogen-bond geometry $\left(A,{ }^{\circ}\right)$

\begin{tabular}{lllll}
\hline$D-\mathrm{H} \cdots A$ & $D-\mathrm{H}$ & $\mathrm{H} \cdots A$ & $D \cdots A$ & $D-\mathrm{H} \cdots A$ \\
\hline $\mathrm{C} 19-\mathrm{H} 19 \cdots \mathrm{N} 2$ & 0.93 & 2.44 & $2.758(3)$ & 100 \\
$\mathrm{C} 23-\mathrm{H} 23 \cdots \mathrm{O} 1$ & 0.93 & 2.21 & $2.832(3)$ & 123 \\
\hline
\end{tabular}

